\title{
BOUNDS ON THE SPEED OF TYPE II BLOW-UP FOR THE ENERGY CRITICAL WAVE EQUATION IN THE RADIAL CASE
}

\author{
JACEK JENDREJ
}

\begin{abstract}
We consider the focusing energy-critical wave equation in space dimension $N \in\{3,4,5\}$ for radial data. We study type II blow-up solutions which concentrate one bubble of energy. It is known that such solutions decompose in the energy space as a sum of the bubble and an asymptotic profile. We prove bounds on the blow-up speed in the case when the asymptotic profile is sufficiently regular. These bounds are optimal in dimension $N=5$. We also prove that if the asymptotic profile is sufficiently regular, then it cannot be strictly negative at the origin.
\end{abstract}

\section{INTRODUCTION}

1.1. Setting of the problem. Let $N \in\{3,4,5\}$ be the dimension of the space. For $\boldsymbol{u}_{0}=\left(u_{0}, \dot{u}_{0}\right) \in$ $\mathcal{E}:=\dot{H}^{1}\left(\mathbb{R}^{N}\right) \times L^{2}\left(\mathbb{R}^{N}\right)$, define the energy functional

$$
E\left(\boldsymbol{u}_{0}\right)=\int \frac{1}{2}\left|\dot{u}_{0}\right|^{2}+\frac{1}{2}\left|\nabla u_{0}\right|^{2}-F\left(u_{0}\right) \mathrm{d} x,
$$

where $F\left(u_{0}\right):=\frac{N-2}{2 N}\left|u_{0}\right|^{\frac{2 N}{N-2}}$. Note that $E\left(\boldsymbol{u}_{0}\right)$ is well-defined due to the Sobolev Embedding Theorem. The differential of $E$ is $\mathrm{D} E\left(\boldsymbol{u}_{0}\right)=\left(-\Delta u_{0}-f\left(u_{0}\right), \dot{u}_{0}\right)$, where $f\left(u_{0}\right)=\left|u_{0}\right|^{\frac{4}{N-2}} u_{0}$.

We consider the Cauchy problem for the energy critical wave equation:

$$
\left\{\begin{aligned}
\partial_{t} \boldsymbol{u}(t) & =J \circ \mathrm{D} E(\boldsymbol{u}(t)) \\
\boldsymbol{u}\left(t_{0}\right) & =\boldsymbol{u}_{0} \in \mathcal{E}
\end{aligned}\right.
$$

Here, $J:=\left(\begin{array}{cc}0 & \mathrm{Id} \\ -\mathrm{Id} & 0\end{array}\right)$ is the natural symplectic structure. This equation is often written in the form

$$
\partial_{t t} u=\Delta u+f(u) \text {. }
$$

Equation (NLW] is locally well-posed in the space $\mathcal{E}$, see for example [1] and 22] (the defocusing case), as well as a complete review of the Cauchy theory in [14. In particular, for any initial data $\boldsymbol{u}_{0} \in \mathcal{E}$ there exists a maximal time of existence $\left(T_{-}, T_{+}\right),-\infty \leq T_{-}<t_{0}<T_{+} \leq+\infty$, and a unique solution $\boldsymbol{u} \in C\left(\left(T_{-}, T_{+}\right) ; \mathcal{E}\right)$. In addition, the energy $E$ is a conservation law. In this paper we always assume that the initial data is radially symmetric. This symmetry is preserved by the flow.

For functions $v \in \dot{H}^{1}, \dot{v} \in L^{2}, \boldsymbol{v}=(v, \dot{v}) \in \mathcal{E}$ and $\lambda>0$, we denote

$$
v_{\lambda}(x):=\frac{1}{\lambda^{(N-2) / 2}} v\left(\frac{x}{\lambda}\right), \quad \dot{v}_{\underline{\lambda}}(x):=\frac{1}{\lambda^{N / 2}} \dot{v}\left(\frac{x}{\lambda}\right), \quad \boldsymbol{v}_{\lambda}(x):=\left(v_{\lambda}, \dot{v}_{\underline{\lambda}}\right) .
$$

A change of variables shows that

$$
E\left(\left(\boldsymbol{u}_{0}\right)_{\lambda}\right)=E\left(\boldsymbol{u}_{0}\right)
$$

Equation (NLW) is invariant under the same scaling. If $\boldsymbol{u}=(u, \dot{u})$ is a solution of (NLW) and $\lambda>0$, then $t \mapsto \boldsymbol{u}\left(\left(t-t_{0}\right) / \lambda\right)_{\lambda}$ is also a solution with initial data $\left(\boldsymbol{u}_{0}\right)_{\lambda}$ at time $t=0$. This is why equation (NLW) is called energy-critical. 
A fundamental object in the study of (NLW) is the family of stationary solutions $\left(u, \partial_{t} u\right)=$ $\pm \boldsymbol{W}_{\lambda}=\left( \pm W_{\lambda}, 0\right)$, where

$$
W(x)=\left(1+\frac{|x|^{2}}{N(N-2)}\right)^{-(N-2) / 2} .
$$

The functions $W_{\lambda}$ are called ground states.

In general the energy $E$ does not control the norm $\|\cdot\|_{\mathcal{E}}$, and indeed this norm can tend to $+\infty$ in finite time, which is referred to as type I blow-up. In odd space dimensions and for superconformal nonlinearities (which includes the energy-critical case) Donninger and Schörkhuber [5, [6] described large sets of initial data leading to this kind of blow-up.

It can also happen that in finite time the solution leaves every compact set of $\mathcal{E}$, the norm $\|\cdot\|_{\mathcal{E}}$ staying bounded, which is referred to as type II blow-up. In dimension $N=3$ in the radial case one of the consequences of the classification result of Duyckaerts, Kenig and Merle [9] is that any blow-up solution is either of type I or of type II. This is unknown in other cases.

A particular type of type II blow-up occurs when the solution $\boldsymbol{u}(t)$ stays close to the family of ground states $\boldsymbol{W}_{\lambda}$ and $\lambda \rightarrow 0$. In this situation we call $\boldsymbol{W}_{\lambda}$ the bubble of energy and we say that $\boldsymbol{u}(t)$ blows up by concentration of one bubble of energy. We have the following fundamental result proved first by Duyckaerts, Kenig and Merle [7] for $N=3$, by the same authors [8] for $N=5$ and by Côte, Kenig, Lawrie and Schlag [4] for $N=4$ :

Theorem ([7], 8], 4]). Let $\boldsymbol{u}(t)$ be a radial solution of (NLW) which blows up at $t=T_{+}$by concentration of one bubble of energy. Then there exist $\boldsymbol{u}_{0}^{*} \in \mathcal{E}$ and $\lambda \in C\left(\left[t_{0}, T_{+}\right),(0,+\infty)\right)$ such that

$$
\lim _{t \rightarrow T_{+}}\left\|\boldsymbol{u}(t)-\boldsymbol{W}_{\lambda(t)}-\boldsymbol{u}_{0}^{*}\right\|_{\mathcal{E}}=0, \quad \lim _{t \rightarrow T_{+}}\left(T_{+}-t\right)^{-1} \lambda(t)=0 .
$$

In this context the function $\boldsymbol{u}_{0}^{*}$ is called the asymptotic profile. Note that in [8] a more general, non-radial version of the above theorem was proved for $N \in\{3,5\}$.

Solutions verifying (1.1) were first constructed in dimension $N=3$ by Krieger, Schlag and Tataru [15], who obtained all possible polynomial blow-up rates $\lambda(t) \sim\left(T_{+}-t\right)^{1+\nu}, \nu>0$. For $N=4$ smooth solutions blowing up at a particular rate were constructed by Hillairet and Raphaël [12]. For $N=5$ the author proved in [13] that for any radially symmetric asymptotic profile $\boldsymbol{u}_{0}^{*} \in H^{4} \times H^{3}$ such that $u_{0}^{*}(0)>0$, there exists a solution $\boldsymbol{u}(t)$ such that (1.1) holds. For these solutions the concentration speed of the bubble is

$$
\lambda(t) \sim u_{0}^{*}(0)^{2}\left(T_{+}-t\right)^{4} .
$$

In the same article, solutions with blow-up rate $\left(T_{+}-t\right)^{1+\nu}$ for $\nu>8$ were constructed, with $\nu$ explicitely related to the asymptotic behaviour of $\boldsymbol{u}_{0}^{*}$ at $x=0$.

1.2. Statement of the results. In the present paper we continue the investigation of the relationship between the behaviour of $\boldsymbol{u}_{0}^{*}$ at $x=0$ and possible blow-up speeds, still in the special case when the asymptotic profile $\boldsymbol{u}_{0}^{*}$ is sufficiently regular. We prove the following result.

Theorem 1. Let $N \in\{3,4,5\}$ and $s>\frac{N-2}{2}, s \geq 1$. Let $\boldsymbol{u}_{0}^{*}=\left(u_{0}^{*}, \dot{u}_{0}^{*}\right) \in H^{s+1} \times H^{s}$ be a radial function. Suppose that $\boldsymbol{u}$ is a radial solution of (NLW) such that

$$
\lim _{t \rightarrow T_{+}}\left\|\boldsymbol{u}(t)-\boldsymbol{W}_{\lambda(t)}-\boldsymbol{u}_{0}^{*}\right\|_{\mathcal{E}}=0, \quad \lim _{t \rightarrow T_{+}} \lambda(t)=0, \quad T_{+}<+\infty .
$$

There exists a constant $C>0$ depending on $\boldsymbol{u}_{0}^{*}$ such that:

- if $N \in\{4,5\}$, then for $T_{+}-t$ sufficiently small there holds

$$
\lambda(t) \leq C\left(T_{+}-t\right)^{\frac{4}{6-N}} .
$$


- if $N=3$, then there exists a sequence $t_{n} \rightarrow T_{+}$such that

$$
\lambda\left(t_{n}\right) \leq C\left(T_{+}-t_{n}\right)^{\frac{4}{6-N}} .
$$

Remark 1.1. Let $\boldsymbol{u}^{*}=\left(u^{*}, \dot{u}^{*}\right)$ be the solution of (NLW) such that $\boldsymbol{u}^{*}\left(T_{+}\right)=\boldsymbol{u}_{0}^{*}$ and suppose that $0 \in \operatorname{supp} \boldsymbol{u}_{0}^{*}$. We will prove that there exists a universal constant $C_{0}$ such that in the above theorem one can take

$$
C=C_{0}\left\|u^{*}\right\|_{L^{\infty}\left(\left(T_{+}-\rho, T_{+}\right) \times B(0, \rho)\right)}^{\frac{2}{6-N}},
$$

where $\rho>0$ is arbitrary and $B(0, \rho)$ is the ball of centre 0 and radius $\rho$ in $\mathbb{R}^{N}$. Notice that $u^{*} \in L^{\infty}\left(\left(T_{+}-\rho, T_{+}\right) \times \mathbb{R}^{N}\right)$ by Appendix $\mathrm{A}$ and the Sobolev Embedding Theorem.

If $0 \notin \operatorname{supp} \boldsymbol{u}_{0}^{*}$, then blow-up cannot occur, as follows from the classification of solutions of (NLW) at energy level $E(\boldsymbol{W})$ by Duyckaerts and Merle [10].

Remark 1.2. In the case $N=3$ we will prove that for $T_{+}-t$ small enough there holds

$$
\int_{t}^{T_{+}} \frac{\mathrm{d} \tau}{\sqrt{\lambda(\tau)}} \geq \frac{3}{\sqrt{C}}\left(T_{+}-t\right)^{\frac{1}{3}}
$$

which immediately implies (1.5).

If we assume that $\boldsymbol{u}^{*} \in H^{3} \times H^{2}$, then (1.4) holds also in the case $N=3$, see Remark 2.13. I believe that the proof of (1.5) given here could be adapted to cover the case $1>s>\frac{1}{2}$.

Remark 1.3. In dimension $N=5$ the bound (1.4) is optimal, see (1.2). It is not clear if the bounds are optimal for $N \in\{3,4\}$, due to slow decay of the bubble.

Remark 1.4. A natural problem is to determine sharp bounds for the blow-up speed in the case of less regular $\boldsymbol{u}_{0}^{*}$. The method used in this paper allows to obtain some bounds for example in the case $1 \leq s<\frac{3}{2}$ in dimension $N=5$, but they are not optimal and I will not pursue this direction here.

In the case $u_{0}^{*}(0)=0$ one could obtain various bounds depending on the asymptotics of $\boldsymbol{u}_{0}^{*}$ at $x=0$, but this will not be considered in the present paper. Along the same line, one can ask if the sign of $u_{0}^{*}(0)$ is relevant in the case when $u_{0}^{*}(0) \neq 0$. It turns out that it is, but unfortunately our method requires the additional assumption $\boldsymbol{u}_{0}^{*} \in H^{3} \times H^{2}$ :

Theorem 2. Let $N \in\{3,4,5\}$. Let $\boldsymbol{u}_{0}^{*}=\left(u_{0}^{*}, \dot{u}_{0}^{*}\right) \in H^{3} \times H^{2}$ be a radial function such that

$$
u_{0}^{*}(0)<0 .
$$

There exist no radial solutions of (NLW) such that

$$
\lim _{t \rightarrow T_{+}}\left\|\boldsymbol{u}(t)-\boldsymbol{W}_{\lambda(t)}-\boldsymbol{u}_{0}^{*}\right\|_{\mathcal{E}}=0, \quad \lim _{t \rightarrow T_{+}} \lambda(t)=0, \quad T_{+}<+\infty .
$$

Remark 1.5. I expect that Theorems 1 and 2 could be proved by similar methods without the assumption of $\boldsymbol{u}_{0}^{*}$ being radial.

1.3. Related results. The problem of existence of an asymptotic profile at blow-up might be seen as a version of the classical question of asymptotic stability of solitons in the case when finite-time blow-up occurs. Decompositions of type (1.1) in suitable topologies are believed to hold for many models, but establishing this rigourously is a challenging problem. Historically, the study of finite type blow-up in the Hamiltonian setting received the most attention probably in the case of nonlinear Schrödinger equations (NLS). For the mass-critical NLS the conformal invariance leads to explicit blow-up solutions $S(t)$ with the asymptotic profile $u^{*} \equiv 0$. Bourgain and Wang [1 constructed examples of blow-up solutions with $u^{*}$ regular and non-zero, the speed of blow-up however being 
the same as for $S(t)$. This is not a coincidence, as shown by a classification result of Merle and Raphaël [19].

For the critical gKdV equation Martel, Merle and Raphaël [17] proved that if the initial data decays sufficiently fast, then there is only one possible blow-up speed, given by the minimal mass blow-up solution. However, without the decay assumption other blow-up speeds are possible, as shown by the same authors in [18].

These are the main two examples of the heuristic principle that the size of the interaction of the bubble with the rest of the solution influences or even determines the speed of blow-up. In the present paper we try to investigate this phenomenon in the energy-critical setting.

Finally, let us mention that the problem of understanding the possible blow-up speeds is not limited to type II blow-up for critical equations. For example, for the subconformal and conformal NLW this was considered in the works of Merle and Zaag [20], 21.

1.4. Outline of the proof. Our proofs of Theorems 1 and 2 are based on the following computation that we present here formally.

Let $\boldsymbol{u}:\left[t_{0} ; T_{+}\right) \rightarrow \mathcal{E}$ be a solution of (NLW) which satisfies (1.1). At blow-up time, the energy of the bubble is completely decoupled from the energy of the asymptotic profile, hence

$$
E(\boldsymbol{u})=E\left(\boldsymbol{u}_{0}^{*}\right)+E\left(\boldsymbol{W}_{\lambda}\right)=E\left(\boldsymbol{u}_{0}^{*}\right)+E(\boldsymbol{W}) .
$$

Let $\boldsymbol{u}^{*}$ be the solution of (NLW) with the initial data $\boldsymbol{u}^{*}\left(T_{+}\right)=\boldsymbol{u}_{0}^{*}$. Decompose $\boldsymbol{u}(t)=\boldsymbol{W}_{\lambda(t)}+$ $\boldsymbol{u}^{*}(t)+\boldsymbol{g}(t)$. The modulation parameter $\lambda$ is determined by a suitable orthogonality condition, and a standard procedure shows that $\left|\lambda^{\prime}(t)\right| \lesssim\|\boldsymbol{g}(t)\|_{\mathcal{E}}$.

From the Taylor formula we obtain

$$
E(\boldsymbol{u})=E\left(\boldsymbol{u}^{*}+\boldsymbol{W}_{\lambda}\right)+\left\langle\mathrm{D} E\left(\boldsymbol{u}^{*}+\boldsymbol{W}_{\lambda}\right), \boldsymbol{g}\right\rangle+\frac{1}{2}\left\langle\mathrm{D}^{2} E\left(\boldsymbol{u}^{*}+\boldsymbol{W}_{\lambda}\right) \boldsymbol{g}, \boldsymbol{g}\right\rangle+O\left(\|\boldsymbol{g}\|_{\mathcal{E}}^{3}\right) .
$$

Step 1. An explicit key computation shows that

$$
E\left(\boldsymbol{u}^{*}+\boldsymbol{W}_{\lambda}\right)-E\left(\boldsymbol{u}^{*}\right)-E(\boldsymbol{W}) \gtrsim-u_{0}^{*}(0) \lambda^{\frac{N-2}{2}} .
$$

It is clear that the sign of $u_{0}^{*}(0)$ is decisive.

Step 2. Near blow-up time $\boldsymbol{u}^{*}$ weakly interacts with $\boldsymbol{W}_{\lambda}$ and $\mathrm{D} E\left(\boldsymbol{W}_{\lambda}\right)=0$. This allows to replace $\left\langle\mathrm{D} E\left(\boldsymbol{u}^{*}+\boldsymbol{W}_{\lambda}\right), \boldsymbol{g}\right\rangle$ by $\left\langle\mathrm{D} E\left(\boldsymbol{u}^{*}\right), \boldsymbol{g}\right\rangle$. Using the Hamiltonian structure it is seen that this quantity is, at first order in $\boldsymbol{g}$, a conservation law. Estimating some error terms we conclude that this term can be neglected.

Step 3. Let us suppose for a moment that $\mathrm{D}^{2} E(\boldsymbol{W})$ is a coercive functional in the sense that $\left\langle\mathrm{D}^{2} E\left(\boldsymbol{u}^{*}+\boldsymbol{W}_{\lambda}\right) \boldsymbol{g}, \boldsymbol{g}\right\rangle \gtrsim\|\boldsymbol{g}\|_{\mathcal{E}}^{2}$. Using (1.7) and the two preceding steps we find $\left|\lambda^{\prime}\right|^{2} \lesssim\|\boldsymbol{g}\|_{\mathcal{E}}^{2} \lesssim$ $u_{0}^{*}(0) \lambda^{\frac{N-2}{2}}$. In the case $u_{0}^{*}(0)<0$ this is contradictory, and in the case $u_{0}^{*}(0)>0$ the conclusion follows by integrating the differential inequality for $\lambda$.

Strictly speaking, $\mathrm{D}^{2} E(\boldsymbol{W})$ is not a coercive functional, and much of the proof is devoted to controlling the negative directions, which are related to the eigendirections of the flow linearized around $\boldsymbol{W}$. Clarifying the second step above is another major technical difficulty of this paper.

1.5. Acknowledgements. This paper has been prepared as a part of my $\mathrm{Ph}$. $\mathrm{D}$. under supervision of Y. Martel and F. Merle. I would like to thank my supervisors for their constant support and many helpful discussions. The author has been supported by the ERC grant 291214 BLOWDISOL.

1.6. Notation. We introduce the inifinitesimal generators of scale change

$$
\Lambda_{s}:=\left(\frac{N}{2}-s\right)+x \cdot \nabla \text {. }
$$


For $s=1$ we omit the subscript and write $\Lambda=\Lambda_{1}$. We denote $\Lambda_{\mathcal{E}}, \Lambda_{\mathcal{F}}$ and $\Lambda_{\mathcal{E}^{*}}$ the inifinitesimal generators of the scaling which is critical for a given norm, that is

$$
\Lambda_{\mathcal{E}}=\left(\Lambda, \Lambda_{0}\right), \quad \Lambda_{\mathcal{F}}=\left(\Lambda_{0}, \Lambda_{-1}\right), \quad \Lambda_{\mathcal{E}^{*}}=\left(\Lambda_{-1}, \Lambda_{0}\right)
$$

The dimension of the space will be denoted $N$. The domain of the function spaces is always $\mathbb{R}^{N}$. We introduce the following notation for some frequently used function spaces: $X^{s}:=\dot{H}^{s+1} \cap \dot{H}^{1}$ for $s \geq 0, \mathcal{E}:=\dot{H}^{1} \times L^{2}, \mathcal{F}:=L^{2} \times \dot{H}^{-1}$. The bracket $\langle\cdot, \cdot\rangle$ denotes the distributional pairing and the scalar product in the spaces $L^{2}, L^{2} \times L^{2}$. Notice that $\mathcal{E}^{*} \simeq \dot{H}^{-1} \times L^{2}$ through the natural isomorphism induced by $\langle\cdot, \cdot\rangle$.

For a function space $\mathcal{A}, O_{\mathcal{A}}(m)$ denotes any $a \in \mathcal{A}$ such that $\|a\|_{\mathcal{A}} \leq \mathrm{Cm}$ for some constant $C>0$. For positive quantities $m_{1}$ and $m_{2}$ we write $m_{1} \lesssim m_{2}$ for $m_{1}=O\left(m_{2}\right)$ and $m_{1} \sim m_{2}$ for $m_{1} \lesssim m_{2} \lesssim m_{1}$. We denote $B_{\mathcal{A}}\left(x_{0}, \delta\right)$ the open ball of center $x_{0}$ and radius $\delta$ in the space $\mathcal{A}$. If $\mathcal{A}$ is not specified, it means that $\mathcal{A}=\mathbb{R}$.

\section{THE PROOFS}

2.1. Properties of the linearized operator. Linearizing $-\Delta u-f(u)$ around $W, u=W+g$, we obtain a Schrödinger operator

$$
L g=\left(-\Delta-f^{\prime}(W)\right) g
$$

Notice that $L(\Lambda W)=\left.\frac{\mathrm{d}}{\mathrm{d} \lambda}\right|_{\lambda=1}\left(-\Delta W_{\lambda}-f\left(W_{\lambda}\right)\right)=0$. It is known that $L$ has exactly one strictly negative simple eigenvalue which we denote $-\nu^{2}$ (we take $\nu>0$ ). We denote the corresponding positive eigenfunction $\mathcal{Y}$, normalized so that $\|\mathcal{Y}\|_{L^{2}}=1$. By elliptic regularity $\mathcal{Y}$ is smooth and by Agmon estimates it decays exponentially. Self-adjointness of $L$ implies that

$$
\langle\mathcal{Y}, \Lambda W\rangle=0 .
$$

We define

$$
\mathcal{Y}^{-}:=\left(\frac{1}{\nu} \mathcal{Y},-\mathcal{Y}\right), \quad \mathcal{Y}^{+}:=\left(\frac{1}{\nu} \mathcal{Y}, \mathcal{Y}\right), \quad \alpha^{-}:=\frac{1}{2}(\nu \mathcal{Y},-\mathcal{Y}), \quad \alpha^{+}:=\frac{1}{2}(\nu \mathcal{Y}, \mathcal{Y})
$$

We have $J \circ \mathrm{D}^{2} E(\boldsymbol{W})=\left(\begin{array}{cc}0 & \mathrm{Id} \\ -L & 0\end{array}\right)$. A short computation shows that

$$
J \circ \mathrm{D}^{2} E(\boldsymbol{W}) \mathcal{Y}^{-}=-\nu \mathcal{Y}^{-}, \quad J \circ \mathrm{D}^{2} E(\boldsymbol{W}) \mathcal{Y}^{+}=\nu \mathcal{Y}^{+}
$$

and

$$
\left\langle\alpha^{-}, J \circ \mathrm{D}^{2} E(\boldsymbol{W}) \boldsymbol{g}\right\rangle=-\nu\left\langle\alpha^{-}, \boldsymbol{g}\right\rangle, \quad\left\langle\alpha^{+}, J \circ \mathrm{D}^{2} E(\boldsymbol{W}) \boldsymbol{g}\right\rangle=\nu\left\langle\alpha^{+}, \boldsymbol{g}\right\rangle, \quad \forall \boldsymbol{g} \in \mathcal{E} .
$$

Notice that $\left\langle\alpha^{-}, \mathcal{Y}^{-}\right\rangle=\left\langle\alpha^{+}, \mathcal{Y}^{+}\right\rangle=1$ and $\left\langle\alpha^{-}, \mathcal{Y}^{+}\right\rangle=\left\langle\alpha^{+}, \mathcal{Y}^{-}\right\rangle=0$

The rescaled versions of these objects are

$$
\mathcal{Y}_{\lambda}^{-}:=\left(\frac{1}{\nu} \mathcal{Y}_{\lambda},-\mathcal{Y}_{\underline{\lambda}}\right), \quad \mathcal{Y}_{\lambda}^{+}:=\left(\frac{1}{\nu} \mathcal{Y}_{\lambda}, \mathcal{Y}_{\underline{\lambda}}\right), \quad \alpha_{\lambda}^{-}:=\frac{1}{2}\left(\frac{\nu}{\lambda} \mathcal{Y}_{\underline{\lambda}},-\mathcal{Y}_{\underline{\lambda}}\right), \quad \alpha_{\lambda}^{+}:=\frac{1}{2}\left(\frac{\nu}{\lambda} \mathcal{Y}_{\underline{\lambda}}, \mathcal{Y}_{\underline{\lambda}}\right) .
$$

The scaling is chosen so that $\left\langle\alpha_{\lambda}^{-}, \mathcal{Y}_{\lambda}^{-}\right\rangle=\left\langle\alpha_{\lambda}^{+}, \mathcal{Y}_{\lambda}^{+}\right\rangle=1$. We have

$$
J \circ \mathrm{D}^{2} E\left(\boldsymbol{W}_{\lambda}\right) \mathcal{Y}_{\lambda}^{-}=-\frac{\nu}{\lambda} \mathcal{Y}_{\lambda}^{-}, \quad J \circ \mathrm{D}^{2} E\left(\boldsymbol{W}_{\lambda}\right) \mathcal{Y}_{\lambda}^{+}=\frac{\nu}{\lambda} \mathcal{Y}_{\lambda}^{+}
$$

and

$$
\left\langle\alpha_{\lambda}^{-}, J \circ \mathrm{D}^{2} E\left(\boldsymbol{W}_{\lambda}\right) \boldsymbol{g}\right\rangle=-\frac{\nu}{\lambda}\left\langle\alpha_{\lambda}^{-}, \boldsymbol{g}\right\rangle, \quad\left\langle\alpha_{\lambda}^{+}, J \circ \mathrm{D}^{2} E\left(\boldsymbol{W}_{\lambda}\right) \boldsymbol{g}\right\rangle=\frac{\nu}{\lambda}\left\langle\alpha_{\lambda}^{+}, \boldsymbol{g}\right\rangle, \quad \forall \boldsymbol{g} \in \mathcal{E} .
$$


Let $\mathcal{Z}$ be a $C_{0}^{\infty}$ function such that

$$
\langle\mathcal{Z}, \Lambda W\rangle>0, \quad\langle\mathcal{Z}, \mathcal{Y}\rangle=0
$$

(the first condition is the essential one and the second allows to simplify some computations). We recall the following result.

Proposition 2.1 ([13, Lemma 6.1], [10, Proposition 5.5]). There exists a constant $c_{L}>0$ such that

$$
v \in \dot{H}^{1} \text { radial }, \quad\langle\mathcal{Y}, v\rangle=\langle\mathcal{Z}, v\rangle=0 \quad \Rightarrow \quad \frac{1}{2}\langle v, L v\rangle \geq c_{L}\|v\|_{\dot{H}^{1}}^{2} .
$$

Lemma 2.2. There exists a constant $c>0$ such that if $\left\|\boldsymbol{V}-\boldsymbol{W}_{\lambda}\right\|_{\mathcal{E}}<c$, then for all $\boldsymbol{g} \in \mathcal{E}$ such that $\left\langle\mathcal{Z}_{\underline{\lambda}}, g\right\rangle=0$ there holds

$$
\frac{1}{2}\left\langle\mathrm{D}^{2} E(\boldsymbol{V}) \boldsymbol{g}, \boldsymbol{g}\right\rangle+2\left(\left\langle\alpha_{\lambda}^{-}, \boldsymbol{g}\right\rangle^{2}+\left\langle\alpha_{\lambda}^{+}, \boldsymbol{g}\right\rangle^{2}\right) \gtrsim\|\boldsymbol{g}\|_{\mathcal{E}}^{2}
$$

Proof. We have

$$
\left\langle\mathrm{D}^{2} E(\boldsymbol{V}) \boldsymbol{g}, \boldsymbol{g}\right\rangle=\left\langle\mathrm{D}^{2} E\left(\boldsymbol{W}_{\lambda}\right) \boldsymbol{g}, \boldsymbol{g}\right\rangle+\int\left(f^{\prime}(V)-f^{\prime}\left(W_{\lambda}\right)\right)|g|^{2} \mathrm{~d} x .
$$

By Hölder, the last integral is $\lesssim c\|\boldsymbol{g}\|_{\mathcal{E}}^{2}$, hence it suffices to prove the lemma with $\boldsymbol{V}=\boldsymbol{W}_{\lambda}$. Without loss of generality we can assume that $\lambda=1$. We will show the following stronger inequality:

$$
\frac{1}{2}\left\langle\mathrm{D}^{2} E(\boldsymbol{W}) \boldsymbol{g}, \boldsymbol{g}\right\rangle+2\left\langle\alpha^{-}, \boldsymbol{g}\right\rangle \cdot\left\langle\alpha^{+}, \boldsymbol{g}\right\rangle \geq c_{L}\left\|\boldsymbol{g}-\left\langle\alpha^{-}, \boldsymbol{g}\right\rangle \mathcal{Y}^{-}-\left\langle\alpha^{+}, \boldsymbol{g}\right\rangle \mathcal{Y}^{+}\right\|_{\mathcal{E}}^{2} .
$$

Let $a^{-}=\left\langle\alpha^{-}, \boldsymbol{g}\right\rangle, a^{+}=\left\langle\alpha^{+}, \boldsymbol{g}\right\rangle$ and decompose $\boldsymbol{g}=a^{-} \mathcal{Y}^{-}+a^{+} \mathcal{Y}^{+}+\boldsymbol{k}$, so that $\left\langle\alpha^{-}, \boldsymbol{k}\right\rangle=$ $\left\langle\alpha^{+}, \boldsymbol{k}\right\rangle=0$. From $\langle\mathcal{Z}, \mathcal{Y}\rangle=0$ we deduce $\langle\mathcal{Z}, k\rangle=0$. We have $g=\frac{a^{-}+a^{+}}{\nu} \mathcal{Y}+k$ and $\dot{g}=$ $\left(-a^{-}+a^{+}\right) \mathcal{Y}+\dot{k}$, hence

$$
\begin{aligned}
\frac{1}{2}\left\langle\mathrm{D}^{2} E(\boldsymbol{W}) \boldsymbol{g}, \boldsymbol{g}\right\rangle & =\frac{1}{2}\left\langle\frac{a^{-}+a^{+}}{\nu} \mathcal{Y}+k,-\left(a^{-}+a^{+}\right) \nu \mathcal{Y}+L k\right\rangle \\
& +\frac{1}{2}\left\langle\left(-a^{-}+a^{+}\right) \mathcal{Y}+\dot{k},\left(-a^{-}+a^{+}\right) \mathcal{Y}+\dot{k}\right\rangle \\
& =-\frac{1}{2}\left(a^{-}+a^{+}\right)^{2}\langle\mathcal{Y}, \mathcal{Y}\rangle-\left(a^{-}+a^{+}\right) \nu\langle\mathcal{Y}, k\rangle+\frac{1}{2}\langle k, L k\rangle \\
& +\frac{1}{2}\left(-a^{-}+a^{+}\right)^{2}\langle\mathcal{Y}, \mathcal{Y}\rangle+\left(-a^{-}+a^{+}\right)\langle\mathcal{Y}, \dot{k}\rangle+\frac{1}{2}\langle\dot{k}, \dot{k}\rangle \\
& =-2 a^{-} a^{+}\langle\mathcal{Y}, \mathcal{Y}\rangle-2 a^{-}\left\langle\alpha^{+}, \boldsymbol{k}\right\rangle-2 a^{+}\left\langle\alpha^{-}, \boldsymbol{k}\right\rangle+\frac{1}{2}(\langle k, L k\rangle+\langle\dot{k}, \dot{k}\rangle) \\
& =-2 a^{-} a^{+}+\frac{1}{2}\left\langle\mathrm{D}^{2} E(\boldsymbol{W}) \boldsymbol{k}, \boldsymbol{k}\right\rangle .
\end{aligned}
$$

Invoking Proposition 2.1 finishes the proof of (2.3).

2.2. Modulation. Recall that $X^{s}:=\dot{H}^{s+1} \cap \dot{H}^{1}$. Let $\boldsymbol{u}_{0}^{*} \in X^{s} \times H^{s}, T_{+} \in \mathbb{R}$ and let $\boldsymbol{u}^{*}$ be the solution of (NLW) with initial data $\boldsymbol{u}^{*}\left(T_{+}\right)=\boldsymbol{u}_{0}^{*}$. Without loss of generality we will assume that $\frac{N-2}{2}<s \leq 2$. For fixed $\rho>0$ we denote

$$
c^{*}:=\left\|u^{*}\right\|_{L^{\infty}\left(\left(T_{+}-\rho, T_{+}\right) \times B(0, \rho)\right)} .
$$

We can assume that $c^{*}>0$ (otherwise there is no blow-up, cf. Remark 1.1). Note that because of finite speed of propagation, we can also assume that $\left\|\boldsymbol{u}^{*}(t)\right\|_{\mathcal{E}}$ is smaller than any fixed strictly positive constant and that $\left\|u^{*}(t)\right\|_{L^{\infty}} \leq 2 c^{*}$ for $t$ close to $T_{+}$. 
Because of a slow decay of $W$, we will introduce compactly supported approximations of $W_{\lambda}$. Let

$$
R:=\left(c_{0} \cdot c^{*}\right)^{\frac{1}{-N+2}},
$$

where $c_{0}>0$ is a small universal constant to be chosen later.

We denote

$$
V(\lambda)(x):= \begin{cases}W_{\lambda}(x)-\zeta(\lambda) & \text { for }|x| \leq R \sqrt{\lambda} \\ 0 & \text { for }|x| \geq R \sqrt{\lambda}\end{cases}
$$

where

$$
\zeta(\lambda):=W_{\lambda}(R \sqrt{\lambda})=\frac{1}{\lambda^{\frac{N-2}{2}}}\left(1+\frac{R^{2}}{N(N-2) \lambda}\right)^{-\frac{N-2}{2}}=\left(\lambda+\frac{R^{2}}{N(N-2)}\right)^{-\frac{N-2}{2}} .
$$

We will also denote

$$
\boldsymbol{V}(\lambda):=(V(\lambda), 0) \in \mathcal{E}
$$

Notice that

$$
\partial_{\lambda} V(\lambda)(x)= \begin{cases}-(\Lambda W)_{\underline{\lambda}}(x)-\zeta^{\prime}(\lambda) & \text { for }|x|<R \sqrt{\lambda} \\ 0 & \text { for }|x|>R \sqrt{\lambda}\end{cases}
$$

Lemma 2.3. Let $s>\frac{N-2}{2}$ and $s \geq 1$. The following estimates are true with universal constants:

$$
\begin{aligned}
\left\|V(\lambda)-W_{\lambda}\right\|_{\dot{H}^{1}} & \lesssim R^{\frac{-N+2}{2} \lambda^{\frac{N-2}{4}},} \\
\left\|V(\lambda)-W_{\lambda}\right\|_{L^{\infty}} & \lesssim R^{-N+2}, \\
\left\|\partial_{\lambda} V(\lambda)+\Lambda W_{\underline{\lambda}}\right\|_{L^{\infty}(|x|<R \sqrt{\lambda})} & \lesssim R^{-N}, \\
\left\|\partial_{\lambda} V(\lambda)\right\|_{L^{\frac{2 N}{N+2}}} & \lesssim R^{\frac{6-N}{2}} \lambda^{\frac{N-2}{4}}, \\
\left\|\partial_{\lambda} V(\lambda)\right\|_{H^{1-s}} & \ll \lambda^{\frac{N-4}{2}} \quad \text { as } \lambda \rightarrow 0 .
\end{aligned}
$$

Proof. To prove (2.5), we write

$$
\begin{aligned}
\left\|V(\lambda)-W_{\lambda}\right\|_{\dot{H}^{1}}^{2} & =\int_{|x| \geq R \sqrt{\lambda}}\left|\nabla W_{\lambda}\right|^{2} \mathrm{~d} x=\int_{|x| \geq R / \sqrt{\lambda}}\|\nabla W\|^{2} \mathrm{~d} x \\
& \lesssim \int_{R / \sqrt{\lambda}}^{+\infty} r^{-2 N+2} \cdot r^{N-1} \mathrm{~d} r \sim(R / \sqrt{\lambda})^{-N+2} .
\end{aligned}
$$

We see that $\zeta(\lambda) \sim R^{-(N-2)}$ and $\zeta^{\prime}(\lambda) \sim R^{-N}$ when $\lambda$ is small, which proves (2.6) and (2.7).

On the support of $\partial_{\lambda} V(\lambda)$ there holds $\left|\partial_{\lambda} V(\lambda)(x)\right| \lesssim \lambda^{\frac{N-4}{2}}|x|^{-N+2}$, hence

$$
\begin{aligned}
\left\|\partial_{\lambda} V(\lambda)\right\|_{L^{\frac{2 N}{N+2}}}^{\frac{2 N}{N+2}} & \lesssim \int_{0}^{R \sqrt{\lambda}} \lambda^{\frac{N-4}{2} \cdot \frac{2 N}{N+2}} r^{(-N+2) \frac{2 N}{N+2}} r^{N-1} \mathrm{~d} r \\
& =\lambda^{\frac{N^{2}-4 N}{N+2}} \int_{0}^{R \sqrt{\lambda}} r^{\frac{-N^{2}+5 N-2}{N+2}} \mathrm{~d} r=R^{\frac{N(6-N)}{N+2}} \lambda^{\frac{N(N-2)}{2(N+2)}} .
\end{aligned}
$$

This proves (2.8).

We will check (2.9) separately in each dimension. For $N=3$ we have $\left|\partial_{\lambda} V(\lambda)(x)\right| \lesssim \lambda^{-\frac{1}{2}}|x|^{-1}$ and $\left\||x|^{-1}\right\|_{L^{2}(|x| \leq R \sqrt{\lambda})} \ll 1$. For $N=4$ we have $\left|\partial_{\lambda} V(\lambda)(x)\right| \lesssim|x|^{-2}$. We suppose $s>1$, hence there exists $q \in(1,2)$ such that $L^{q} \subset H^{1-s}$ and it is easy to check that $\left\||x|^{-2}\right\|_{L^{q}(|x| \leq R \sqrt{\lambda})} \ll 1$. 
Finally for $N=5$ we have $\left|\partial_{\lambda} V(\lambda)(x)\right| \lesssim \lambda^{\frac{1}{2}}|x|^{-3}$. There exists $q \in\left(1, \frac{5}{3}\right)$ such that $L^{q} \subset H^{1-s}$ and it is easy to check that $\left\||x|^{-3}\right\|_{L^{q}(|x| \leq R \sqrt{\lambda})} \ll 1$.

Note that $\zeta(\lambda) \sim c_{0} c^{*}$, which means that the cut-off is made at a radius $r=R \sqrt{\lambda}$ such that $W_{\lambda}(r) \sim c_{0} u^{*}(t, r)$.

For the next lemma we will need the following version of the Implicit Function Theorem. It is obtained directly from standard proofs of the usual version, see for example [2, Section 2.2].

Lemma 2.4. Suppose that $X, Y$ and $Z$ are Banach spaces, $x_{0} \in X, y_{0} \in Y, \rho, \eta>0$ and $\Phi: B\left(x_{0}, \rho\right) \times B\left(y_{0}, \eta\right) \rightarrow Z$ is continuous in $x$ and continuously differentiable in $y, \Phi\left(x_{0}, y_{0}\right)=0$ and $\mathrm{D}_{y} \Phi\left(x_{0}, y_{0}\right)=: L_{0}$ has a bounded inverse. Suppose that

$$
\begin{aligned}
\left\|L_{0}-\mathrm{D}_{y} \Phi(x, y)\right\|_{Z} & \leq \frac{1}{3}\left\|L_{0}^{-1}\right\|_{\mathscr{L}(Z, Y)}^{-1} & & \text { for }\left\|x-x_{0}\right\|_{X}<\rho,\left\|y-y_{0}\right\|_{Y}<\eta, \\
\left\|\Phi\left(x, y_{0}\right)\right\|_{Z} & \leq \frac{\eta}{3}\left\|L_{0}^{-1}\right\|_{\mathscr{L}(Z, Y)}^{-1} & & \text { for }\left\|x-x_{0}\right\|_{X}<\rho .
\end{aligned}
$$

Then there exists $y \in C\left(B\left(x_{0}, \rho\right), B\left(y_{0}, \eta\right)\right)$ such that for $x \in B\left(x_{0}, \rho\right), y(x)$ is the unique solution of the equation $\Phi(x, y(x))=0$ in $B\left(y_{0}, \eta\right)$.

Lemma 2.5. There exists $\delta_{0}>0$ and $\lambda_{0}>0$ such that for any $0 \leq \delta \leq \delta_{0}$ and $t_{1}<t_{2}$, if $\boldsymbol{u}:\left(t_{1}, t_{2}\right) \rightarrow \mathcal{E}$ is a solution of (NLW) satisfying for all $t \in\left(t_{1}, t_{2}\right)$ :

$$
\left\|\boldsymbol{u}(t)-\boldsymbol{u}^{*}(t)-\boldsymbol{W}_{\widetilde{\lambda}(t)}\right\|_{\mathcal{E}} \leq \delta, \quad 0<\widetilde{\lambda}(t)<\lambda_{0}
$$

then there exists a unique function $\lambda(t) \in C^{1}\left(\left(t_{1}, t_{2}\right),(0,+\infty)\right)$ such that

$$
\boldsymbol{g}(t):=\boldsymbol{u}(t)-\boldsymbol{u}^{*}(t)-\boldsymbol{V}(\lambda(t))
$$

satisfies for all $t \in\left(t_{1}, t_{2}\right)$ :

$$
\begin{aligned}
\left\langle\mathcal{Z}_{\underline{\lambda(t)}}, g(t)\right\rangle & =0, \\
\|\boldsymbol{g}(t)\|_{\mathcal{E}} & \lesssim \delta+\widetilde{\lambda}(t)^{\frac{N-2}{4}}, \\
|\lambda(t) / \widetilde{\lambda}(t)-1| & \lesssim \delta \\
\left|\lambda^{\prime}(t)\right| & \lesssim\|\boldsymbol{g}(t)\|_{\mathcal{E} .}
\end{aligned}
$$

Proof. We will first show that for $t_{0} \in\left(t_{1}, t_{2}\right)$ fixed there exists a unique $\lambda\left(t_{0}\right)$ such that (2.12), (2.13) and (2.14) hold at $t=t_{0}$. The proof is standard, see for example [16, Proposition 1].

Denote $\boldsymbol{v}_{0}:=\boldsymbol{u}\left(t_{0}\right)-\boldsymbol{u}^{*}\left(t_{0}\right)$ and $\widetilde{l}_{0}:=\log \left(\widetilde{\lambda}\left(t_{0}\right)\right)$ (it will be convenient to consider $\widetilde{\lambda}\left(t_{0}\right)$ and $\lambda\left(t_{0}\right)$ in the logarithmic scale). We define the following functional:

$$
\Phi: \mathcal{E} \times \mathbb{R} \rightarrow \mathbb{R}, \quad \Phi(\boldsymbol{v} ; l):=\left\langle\mathrm{e}^{-l} \mathcal{Z}_{\underline{\mathrm{e}^{l}}}, v-V\left(\mathrm{e}^{l}\right)\right\rangle .
$$

We have

$$
\partial_{l} \Phi(\boldsymbol{v} ; l)=-\left\langle\underline{\mathcal{Z}}_{\underline{\underline{\mathrm{e}}}}, \partial_{\lambda} V\left(\mathrm{e}^{l}\right)\right\rangle-\left\langle\mathrm{e}^{-l} \Lambda_{-1} \underline{\mathcal{Z}}_{\underline{\mathrm{e}}}, v-V\left(\mathrm{e}^{l}\right)\right\rangle .
$$

We apply Lemma 2.4 with $x_{0}=\boldsymbol{V}\left(\widetilde{\lambda}\left(t_{0}\right)\right)$ and $y_{0}=\widetilde{l}_{0}$. It is easily checked that the assumptions hold if $\delta$ is small and $\eta=C \delta$, with a large constant $C$. Take $\lambda\left(t_{0}\right)=\mathrm{e}^{l_{0}}$, where $l_{0}$ is the solution of $\Phi\left(\boldsymbol{v}_{0} ; l_{0}\right)=0$ given by Lemma 2.4. Directly from the definition of $\Phi$ we obtain (2.12). The inequality $\left|l_{0}-\widetilde{l}_{0}\right| \leq \eta=C \delta$ is equivalent to (2.14), which in turn implies

$$
\left\|W_{\widetilde{\lambda}\left(t_{0}\right)}-W_{\lambda\left(t_{0}\right)}\right\|_{\dot{H}^{1}} \lesssim \delta
$$

From the definition of $\boldsymbol{g}$ and (2.10) we have

$$
\|\boldsymbol{g}\| \leq \delta+\left\|W_{\widetilde{\lambda}\left(t_{0}\right)}-W_{\lambda\left(t_{0}\right)}\right\|_{\dot{H}^{1}}+\left\|W_{\lambda\left(t_{0}\right)}-V\left(\lambda\left(t_{0}\right)\right)\right\|_{\dot{H}^{1}}
$$


so (2.13) follows from (2.16) and (2.5).

For each $t_{0} \in\left(t_{1}, t_{2}\right)$ we have defined $\lambda\left(t_{0}\right)$. It remains to show that $\lambda(t)$ is a $C^{1}$ function and that (2.15) holds. One way is to use a regularization procedure as in [16]. Here we give a different argument, which might be simpler in some cases.

Take $t_{0} \in\left(t_{1}, t_{2}\right)$ and let $l_{0}:=\log \left(\lambda\left(t_{0}\right)\right)$. Denote $\boldsymbol{v}(t):=\boldsymbol{u}(t)-\boldsymbol{u}^{*}(t)$ and define $l:\left(t_{0}-\varepsilon, t_{0}+\varepsilon\right) \rightarrow$ $\mathbb{R}$ as the solution of the differential equation

$$
l^{\prime}(t)=-\left(\partial_{l} \Phi\right)^{-1}\left(\mathrm{D}_{\boldsymbol{v}} \Phi\right) \partial_{t} \boldsymbol{v}(t)
$$

with the initial condition $l\left(t_{0}\right)=l_{0}$. Notice that $\mathrm{D}_{\boldsymbol{v}} \Phi$ is a continuous functional on $\mathcal{F}$, so we can apply it to $\partial_{t} \boldsymbol{v}(t)$.

Using the chain rule we get $\frac{\mathrm{d}}{\mathrm{d} t} \Phi(\boldsymbol{v}(t) ; l(t))=0$ for $t \in\left(t_{0}-\varepsilon, t_{0}+\varepsilon\right)$. By continuity, $\left|l(t)-l_{0}\right|<$ $\eta=C \delta$ in some neighbourhood of $t=t_{0}$. Hence, by the uniqueness part of Lemma 2.4. we get $l(t)=\log \lambda(t)$ in some neighbourhood of $t=t_{0}$. In particular, $\lambda(t)$ is of class $C^{1}$ in some neighbourhood of $t_{0}$.

From (2.11) we obtain the following differential equation for the error term $\boldsymbol{g}$ :

$$
\partial_{t} \boldsymbol{g}=J \circ\left(\mathrm{D} E\left(\boldsymbol{V}(\lambda)+\boldsymbol{u}^{*}+\boldsymbol{g}\right)-\mathrm{D} E\left(\boldsymbol{u}^{*}\right)\right)-\lambda^{\prime} \partial_{\lambda} \boldsymbol{V}(\lambda),
$$

which can also be written in the expanded form

$$
\left\{\begin{array}{l}
\partial_{t} g=\dot{g}-\lambda^{\prime} \partial_{\lambda} V(\lambda), \\
\partial_{t} \dot{g}=\Delta g+\left(f\left(u^{*}+V(\lambda)+g\right)-f\left(u^{*}\right)-f(V(\lambda))\right)+(\Delta V(\lambda)+f(V(\lambda))) .
\end{array}\right.
$$

Differentiating (2.12) and using the first equation in (2.18) we get

$$
\begin{aligned}
0 & =\frac{\mathrm{d}}{\mathrm{d} t}\left\langle\mathcal{Z}_{\underline{\lambda}}, g\right\rangle=-\frac{\lambda^{\prime}}{\lambda}\left\langle\Lambda_{0} \mathcal{Z}_{\underline{\lambda}}, g\right\rangle+\left\langle\mathcal{Z}_{\underline{\lambda}}, \dot{g}-\lambda^{\prime} \partial_{\lambda} V(\lambda)\right\rangle \\
& =\lambda^{\prime}\left(\langle\mathcal{Z}, \Lambda W\rangle-\left\langle\mathcal{Z}_{\underline{\lambda}}, \Lambda W_{\underline{\lambda}}+\partial_{\lambda} V(\lambda)\right\rangle-\left\langle\frac{1}{\lambda} \Lambda_{0} \mathcal{Z}_{\underline{\lambda}}, g\right\rangle\right)+\left\langle\mathcal{Z}_{\underline{\lambda}}, \dot{g}\right\rangle .
\end{aligned}
$$

We assumed that $\langle\mathcal{Z}, \Lambda W\rangle>0$. When $\|\boldsymbol{g}\|_{\mathcal{E}}$ and $\lambda$ are small enough, then

$$
\left|\left\langle\mathcal{Z}_{\underline{\lambda}}, \Lambda W_{\underline{\lambda}}+\partial_{\lambda} V(\lambda)\right\rangle+\left\langle\frac{1}{\lambda} \Lambda_{0} \mathcal{Z}_{\underline{\lambda}}, g\right\rangle\right| \leq \frac{1}{2}\langle\mathcal{Z}, \Lambda W\rangle
$$

(we use (2.7) in order to estimate the first term). This proves (2.15).

If $\boldsymbol{u}(t)$ is a solution of (NLW) satisfying (1.3), then there exists $t_{0}$ such that (2.10) holds for $t \in\left[t_{0}, T_{+}\right)$. It follows from (2.14) that, while proving Theorem 1 without loss of generality we can assume that $\lambda(t)$ is the function given by Lemma 2.5. From (2.13) we obtain that $\|\boldsymbol{g}\|_{\mathcal{E}} \rightarrow 0$ as $t \rightarrow T_{+}$, which is the only information about $\boldsymbol{g}$ used in the sequel. The precise form of the right hand side of (2.13) has no importance. We will prove that (1.4) holds on some interval $\left[t_{0}, T_{+}\right)$with $t_{0}<T_{+}$, with no information about the length of this interval. Each time we state something for $t \in\left[t_{0}, T_{+}\right)$it should be understood that $t_{0}$ is sufficiently close to $T_{+}$.

In the rest of this paper $\lambda(t)$ always stands for the modulation parameter obtained in Lemma 2.5 and $\boldsymbol{g}(t)$ is the function defined by (2.11). We introduce the following notation for the joint size of the error and the interaction:

$$
n(\boldsymbol{g}, \lambda):=\sqrt{\|\boldsymbol{g}\|_{\mathcal{E}}^{2}+c^{*} \lambda^{\frac{N-2}{2}}} .
$$

We will now analyze the stable and unstable directions of the linearized flow. The stable coefficient $a^{-}(t)$ and the unstable coefficient $a^{+}(t)$ are defined as follows:

$$
a^{-}(t):=\left\langle\alpha_{\lambda(t)}^{-}, \boldsymbol{g}(t)\right\rangle, \quad a^{+}(t):=\left\langle\alpha_{\lambda(t)}^{+}, \boldsymbol{g}(t)\right\rangle .
$$

Note that $\left|a^{-}(t)\right| \lesssim\|\boldsymbol{g}\|_{\mathcal{E}}$ and $\left|a^{+}(t)\right| \lesssim\|\boldsymbol{g}\|_{\mathcal{E}}$. 
Lemma 2.6. The functions $a^{-}(t)$ and $a^{+}(t)$ satisfy

$$
\begin{aligned}
\left|\frac{\mathrm{d}}{\mathrm{d} t} a^{-}(t)+\frac{\nu}{\lambda(t)} a^{-}(t)\right| & \lesssim \frac{1}{\lambda(t)} n(\boldsymbol{g}(t), \lambda(t))^{2}, \\
\left|\frac{\mathrm{d}}{\mathrm{d} t} a^{+}(t)-\frac{\nu}{\lambda(t)} a^{+}(t)\right| & \lesssim \frac{1}{\lambda(t)} n(\boldsymbol{g}(t), \lambda(t))^{2} .
\end{aligned}
$$

Proof. We will only prove (2.19); the other estimate can be shown analogously.

Let us rewrite equation (2.18) in the following manner:

$$
\partial_{t} \boldsymbol{g}=J \circ \mathrm{D}^{2} E\left(\boldsymbol{W}_{\lambda}\right) \boldsymbol{g}+\boldsymbol{h},
$$

where

$$
\boldsymbol{h}=\left(\begin{array}{l}
h \\
\dot{h}
\end{array}\right)=\left(\begin{array}{c}
-\lambda^{\prime} \partial_{\lambda} V(\lambda) \\
\left(f\left(u^{*}+V(\lambda)+g\right)-f\left(u^{*}\right)-f(V(\lambda))-f^{\prime}\left(W_{\lambda}\right) g\right)+(\Delta V(\lambda)+f(V(\lambda)))
\end{array}\right) .
$$

Using (2.2) we get

$$
\begin{aligned}
\frac{\mathrm{d}}{\mathrm{d} t} a^{-}(t)+\frac{\nu}{\lambda} a^{-}(t) & =\frac{\mathrm{d}}{\mathrm{d} t}\left\langle\alpha_{\lambda}^{-}, \boldsymbol{g}\right\rangle+\frac{\nu}{\lambda}\left\langle\alpha_{\lambda}^{-}, \boldsymbol{g}\right\rangle \\
& =-\frac{\lambda^{\prime}}{\lambda}\left\langle\Lambda_{\mathcal{E}^{*}} \alpha_{\lambda}^{-}, \boldsymbol{g}\right\rangle+\left\langle\alpha_{\lambda}^{-}, J \circ \mathrm{D}^{2} E\left(\boldsymbol{W}_{\lambda}\right) \boldsymbol{g}\right\rangle+\frac{\nu}{\lambda}\left\langle\alpha_{\lambda}^{-}, \boldsymbol{g}\right\rangle+\left\langle\alpha_{\lambda}^{-}, \boldsymbol{h}\right\rangle \\
& =-\frac{\lambda^{\prime}}{\lambda}\left\langle\Lambda_{\mathcal{E}^{*}} \alpha_{\lambda}^{-}, \boldsymbol{g}\right\rangle+\left\langle\alpha_{\lambda}^{-}, \boldsymbol{h}\right\rangle .
\end{aligned}
$$

The first term is negligible due to (2.15). In order to bound the second term it suffices to check the following inequalities:

$$
\begin{aligned}
\left|\left\langle\mathcal{Y}_{\underline{\lambda}}, \partial_{\lambda} V(\lambda)\right\rangle\right| & \lesssim n(\boldsymbol{g}, \lambda)^{2}, \\
\left|\left\langle\mathcal{Y}_{\lambda},(\Delta V(\lambda)+f(V(\lambda)))\right\rangle\right| & \lesssim n(\boldsymbol{g}, \lambda)^{2}, \\
\left|\left\langle\mathcal{Y}_{\lambda},\left(f\left(u^{*}+V(\lambda)+g\right)-f\left(u^{*}\right)-f(V(\lambda))-f^{\prime}\left(W_{\lambda}\right) g\right)\right\rangle\right| & \lesssim n(\boldsymbol{g}, \lambda)^{2} .
\end{aligned}
$$

The first inequality follows from (2.7) and (2.1), since the region $|x| \geq R \sqrt{\lambda}$ is negligible due to exponential decay of $\mathcal{Y}$.

Notice that $\left|f\left(W_{\lambda}\right)-f(V(\lambda))\right| \lesssim f^{\prime}\left(W_{\lambda}\right)|\cdot| W_{\lambda}-V(\lambda) \mid \lesssim f^{\prime}\left(W_{\lambda}\right) c_{0} c^{*}$, where the last inequality follows from (2.5) and (2.4). Together with the fact that $\Delta\left(W_{\lambda}\right)+f\left(W_{\lambda}\right)=0$ this implies

$$
\begin{aligned}
\left|\left\langle\mathcal{Y}_{\lambda},(\Delta V(\lambda)+f(V(\lambda)))\right\rangle\right| & \lesssim\left|\left\langle\mathcal{Y}_{\lambda}, \Delta\left(W_{\lambda}-V(\lambda)\right)\right\rangle\right|+\left|\left\langle\mathcal{Y}_{\lambda}, f\left(W_{\lambda}\right)-f(V(\lambda))\right\rangle\right| \\
& \lesssim\left(\left\|\Delta Y_{\lambda}\right\|_{L^{1}}+\left\|f^{\prime}\left(W_{\lambda}\right) \mathcal{Y}_{\lambda}\right\|_{L^{1}}\right) c_{0} c^{*} \lesssim c^{*} \lambda^{\frac{N-2}{2}},
\end{aligned}
$$

which proves (2.20).

We will check (2.21) in three small steps. As before, we do not have to worry about the region $|x| \geq R \sqrt{\lambda}$ thanks to the fast decay of $\mathcal{Y}$. First, we have a pointwise bound

$$
\left|f\left(u^{*}+V(\lambda)\right)-f\left(u^{*}\right)-f(V(\lambda))\right| \lesssim f^{\prime}\left(W_{\lambda}\right) \cdot c^{*}+f\left(c^{*}\right)
$$

which implies

$$
\left|\left\langle\mathcal{Y}_{\lambda}, f\left(u^{*}+V(\lambda)\right)-f\left(u^{*}\right)-f(V(\lambda))\right\rangle\right| \lesssim n(\boldsymbol{g}, \lambda)^{2}
$$

Next, we have

$$
\left|f\left(u^{*}+V(\lambda)+g\right)-f\left(u^{*}+V(\lambda)\right)-f^{\prime}\left(u^{*}+V(\lambda)\right) g\right| \lesssim\left|f^{\prime \prime}\left(u^{*}+V(\lambda)\right)\right| \cdot|g|^{2}+f(|g|),
$$

which implies

$$
\left|\left\langle\mathcal{Y}_{\lambda}, f\left(u^{*}+V(\lambda)+g\right)-f\left(u^{*}+V(\lambda)\right)-f^{\prime}\left(u^{*}+V(\lambda)\right) g\right\rangle\right| \lesssim n(\boldsymbol{g}, \lambda)^{2} .
$$


Finally, $\left|f^{\prime}\left(V(\lambda)+u^{*}\right)-f^{\prime}\left(W_{\lambda}\right)\right| \lesssim\left(\left|f^{\prime \prime}\left(W_{\lambda}\right)\right|+\left|f^{\prime \prime}\left(V(\lambda)+u^{*}-W_{\lambda}\right)\right|\right) \cdot\left|V(\lambda)+u^{*}-W_{\lambda}\right| \lesssim\left|f^{\prime \prime}\left(W_{\lambda}\right)\right| c^{*}$. Using Hölder and the fact that $\left\|\mathcal{Y}_{\lambda} \cdot f^{\prime \prime}\left(W_{\lambda}\right)\right\|_{L^{\frac{2 N}{N+2}}} \lesssim \lambda^{\frac{N-2}{2}}$ this implies

$$
\left|\left\langle\mathcal{Y}_{\lambda},\left(f^{\prime}\left(u^{*}+V(\lambda)\right)-f^{\prime}\left(W_{\lambda}\right)\right) g\right\rangle\right| \ll n(\boldsymbol{g}, \lambda)^{2} .
$$

Now (2.21) follows from (2.23), (2.25) and (2.26) and the triangle inequality.

2.3. Coercivity. By the conservation of energy, for all $t \in\left[t_{0}, T_{+}\right)$there holds

$$
E\left(\boldsymbol{V}(\lambda)+\boldsymbol{u}^{*}+\boldsymbol{g}\right)=E(\boldsymbol{W})+E\left(\boldsymbol{u}^{*}\right) .
$$

On the other hand, using the pointwise inequality

$$
\left|F(k+l)-F(k)-f(k) l-\frac{1}{2} f^{\prime}(k) l^{2}\right| \lesssim\left|f^{\prime \prime}(k)\right|\left|l^{3}\right|+|F(l)|, \quad \forall k, l \in \mathbb{R}
$$

we deduce that

Using (2.27) we obtain

$$
\begin{aligned}
E\left(\boldsymbol{V}(\lambda)+\boldsymbol{u}^{*}+\boldsymbol{g}\right) & =E\left(\boldsymbol{V}(\lambda)+\boldsymbol{u}^{*}\right)+\left\langle\mathrm{D} E\left(\boldsymbol{V}(\lambda)+\boldsymbol{u}^{*}\right), \boldsymbol{g}\right\rangle \\
& +\frac{1}{2}\left\langle\mathrm{D}^{2} E\left(\boldsymbol{V}(\lambda)+\boldsymbol{u}^{*}\right) \boldsymbol{g}, \boldsymbol{g}\right\rangle+O\left(\|\boldsymbol{g}\|_{\mathcal{E}}^{3}\right) .
\end{aligned}
$$

$$
\begin{aligned}
\left(E\left(\boldsymbol{V}(\lambda)+\boldsymbol{u}^{*}\right)-E(\boldsymbol{W})-E\left(\boldsymbol{u}^{*}\right)\right) & +\left\langle\mathrm{D} E\left(\boldsymbol{V}(\lambda)+\boldsymbol{u}^{*}\right), \boldsymbol{g}\right\rangle \\
& +\frac{1}{2}\left\langle\mathrm{D}^{2} E\left(\boldsymbol{V}(\lambda)+\boldsymbol{u}^{*}\right) \boldsymbol{g}, \boldsymbol{g}\right\rangle=O\left(\|\boldsymbol{g}\|_{\mathcal{E}}^{3}\right) .
\end{aligned}
$$

We start by computing the size of the first term on the left hand side.

Lemma 2.7. For $T_{+}-t$ small there holds

$$
\left|E\left(\boldsymbol{V}(\lambda)+\boldsymbol{u}^{*}\right)-E(\boldsymbol{W})-E\left(\boldsymbol{u}^{*}\right)\right| \lesssim c^{*} \lambda^{\frac{N-2}{2}} .
$$

In addition, if $u^{*}(0)<0$, then

$$
E\left(\boldsymbol{V}(\lambda)+\boldsymbol{u}^{*}\right)-E(\boldsymbol{W})-E\left(\boldsymbol{u}^{*}\right) \gtrsim c^{*} \lambda^{\frac{N-2}{2}} .
$$

Proof. Integrating by parts we obtain

$$
\begin{aligned}
\int \nabla V(\lambda) \cdot \nabla u^{*} \mathrm{~d} x & =\int_{B(0, R \sqrt{\lambda})} \nabla\left(W_{\lambda}\right) \cdot \nabla u^{*} \mathrm{~d} x \\
& =-\int_{B(0, R \sqrt{\lambda})} \Delta\left(W_{\lambda}\right) \cdot u^{*} \mathrm{~d} x+\int_{S(0, R \sqrt{\lambda})} \partial_{r}\left(W_{\lambda}\right) \cdot u^{*} \mathrm{~d} \sigma \\
& =\int_{B(0, R \sqrt{\lambda})} f\left(W_{\lambda}\right) \cdot u^{*} \mathrm{~d} x+\int_{S(0, R \sqrt{\lambda})} \partial_{r}\left(W_{\lambda}\right) \cdot u^{*} \mathrm{~d} \sigma
\end{aligned}
$$

Developping the energy gives

$$
\begin{aligned}
& E\left(\boldsymbol{V}(\lambda)+\boldsymbol{u}^{*}\right)-E(\boldsymbol{W})-E\left(\boldsymbol{u}^{*}\right)=\int \nabla V(\lambda) \cdot \nabla u^{*} \mathrm{~d} x+\frac{1}{2} \int|\nabla V(\lambda)|^{2}-\left|\nabla\left(W_{\lambda}\right)\right|^{2} \mathrm{~d} x \\
& -\int F\left(V(\lambda)+u^{*}\right)-F\left(W_{\lambda}\right)-F\left(u^{*}\right) \mathrm{d} x \\
& =\int_{S(0, R \sqrt{\lambda})} \partial_{r}\left(W_{\lambda}\right) \cdot u^{*} \mathrm{~d} \sigma+\frac{1}{2} \int|\nabla V(\lambda)|^{2}-\left|\nabla W_{\lambda}\right|^{2} \mathrm{~d} x \\
& -\int F\left(V(\lambda)+u^{*}\right)-F\left(W_{\lambda}\right)-F\left(u^{*}\right)-f(V(\lambda)) \cdot u^{*} \mathrm{~d} x \\
& +\int_{B(0, R \sqrt{\lambda})}\left(f\left(W_{\lambda}\right)-f(V(\lambda))\right) \cdot u^{*} \mathrm{~d} x
\end{aligned}
$$


We will show that all the terms on the right hand side except for the first one are $\lesssim c_{0} c^{*} \lambda^{\frac{N-2}{2}}$, where $c_{0}$ is the small constant in (2.4).

The fact that $\int|\nabla V(\lambda)|^{2}-\left|\nabla W_{\lambda}\right|^{2} \mathrm{~d} x \lesssim c_{0} c^{*} \lambda^{\frac{N-2}{2}}=R^{-N+2} \lambda^{\frac{N-2}{2}}$ follows directly from the proof of (2.5).

We will now show that

$$
\int\left|F\left(V(\lambda)+u^{*}\right)-F(V(\lambda))-F\left(u^{*}\right)-f(V(\lambda)) u^{*}\right| \mathrm{d} x \ll \lambda^{\frac{N-2}{2}} .
$$

To this end, notice first that the integrand equals 0 for $|x| \geq R \sqrt{\lambda}$. In the region $|x| \leq R \sqrt{\lambda}$ we use the pointwise estimate

$$
\left|F\left(V(\lambda)+u^{*}\right)-F(V(\lambda))-F\left(u^{*}\right)-f(V(\lambda)) u^{*}\right| \lesssim f^{\prime}(V(\lambda))\left|u^{*}\right|^{2}+F\left(u^{*}\right) .
$$

The term $F\left(u^{*}\right)$ can be neglected (it is bounded in $L^{\infty}$, so its contribution is at most $\lambda^{\frac{N}{2}} \ll \lambda^{\frac{N-2}{2}}$ ). As for the first term, it is easily checked that

$$
\int_{|x| \leq R \sqrt{\lambda}} f^{\prime}\left(W_{\lambda}\right) \mathrm{d} x=\lambda^{N-2} \int_{|x| \leq R / \sqrt{\lambda}} f^{\prime}(W) \mathrm{d} x \ll \lambda^{\frac{N-2}{2}} .
$$

Next, we show that if $R$ is large enough, then

$$
\int\left|F\left(W_{\lambda}\right)-F(V(\lambda))\right| \mathrm{d} x \lesssim c_{0} c^{*} \lambda^{\frac{N-2}{2}}
$$

In the region $|x| \geq R \sqrt{\lambda}$ from (2.5) and Sobolev embedding we obtain that the contribution is at most $\lambda^{\frac{N}{2}} \ll \lambda^{\frac{N-2}{2}}$. In the region $|x| \leq R \sqrt{\lambda}$ we use the bound

$$
\left|F\left(W_{\lambda}\right)-F(V(\lambda))\right| \lesssim \zeta(\lambda) \cdot\left|f\left(W_{\lambda}\right)\right|+F(\zeta(\lambda)) .
$$

The second term is in $L^{\infty}$, so its integral is at most $O\left(\lambda^{\frac{N}{2}}\right) \ll \lambda^{\frac{N-2}{2}}$. As for the first term, it is easily seen that $\int\left|f\left(W_{\lambda}\right)\right| \mathrm{d} x \lesssim \lambda^{\frac{N-2}{2}}$, and we get the conclusion if we recall that $\zeta(\lambda) \sim c_{0} c^{*}$.

Finally, from (2.31) and the pointwise bound $\left|f(V(\lambda))-f\left(W_{\lambda}\right)\right| \lesssim\left|\zeta(\lambda) f^{\prime}\left(W_{\lambda}\right)\right|+|f(\zeta(\lambda))|$ it follows that

$$
\int_{B(0, R \sqrt{\lambda})}\left|f(V(\lambda))-f\left(W_{\lambda}\right)\right| \cdot\left|u^{*}\right| \mathrm{d} x \ll \lambda^{\frac{N-2}{2}} .
$$

Now consider the first term on the right hand side of (2.30). We have $\partial_{r}\left(W_{\lambda}\right)(R \sqrt{\lambda}) \sim-\lambda^{\frac{N-2}{2}}(R \sqrt{\lambda})^{-N+1}$ and $\left|u^{*}\right| \leq c^{*}$ near the origin, so we get

$$
\left|\int_{S(0, R \sqrt{\lambda})} \partial_{r}\left(W_{\lambda}\right) \cdot u^{*} \mathrm{~d} \sigma\right| \lesssim c^{*} \lambda^{\frac{N-2}{2}} .
$$

In the case $u_{0}^{*}(0)<0$, by continuity if in the definition of $c^{*}$ we choose $\rho$ small enough, then $u^{*}(t, x) \leq-\frac{1}{2} c^{*}$ for $(t, x) \in\left[t_{0}, T_{+}\right) \times B(0, \rho)$. In particular,

$$
\int_{S(0, R \sqrt{\lambda})} \partial_{r}\left(W_{\lambda}\right) \cdot u^{*} \mathrm{~d} \sigma \gtrsim c^{*} \lambda^{\frac{N-2}{2}}
$$

where the constant in this estimate is independent of $c_{0}$. The conclusion follows from (2.30) if $c_{0}$ is chosen small enough.

We will focus at present on the second term on the left hand side of (2.28). In Lemma 2.8 we treat the simpler case $\boldsymbol{u}_{0}^{*} \in X^{2} \times H^{2}$ and in Lemma 2.9 we prove a weaker estimate in the case $\boldsymbol{u}^{*} \in X^{s} \times H^{s}, s>\frac{N-2}{2}, s \geq 1$. 
Lemma 2.8. Suppose that $\boldsymbol{u}_{0}^{*} \in X^{2} \times H^{2}$. Then for $t \in\left[t_{0}, T_{+}\right)$there holds

$$
\left|\left\langle\mathrm{D} E\left(\boldsymbol{V}(\lambda(t))+\boldsymbol{u}^{*}(t)\right), \boldsymbol{g}(t)\right\rangle\right| \lesssim \sqrt{c_{0}} \cdot \sup _{t \leq \tau<T_{+}} n(\boldsymbol{g}(\tau), \lambda(\tau))^{2},
$$

where $c_{0}$ is the small constant in (2.4).

Proof. The proof has two steps. First we will show that

$$
\left|\left\langle\mathrm{D} E\left(\boldsymbol{V}(\lambda(t))+\boldsymbol{u}^{*}(t)\right)-\mathrm{D} E\left(\boldsymbol{u}^{*}(t)\right), \boldsymbol{g}(t)\right\rangle\right| \lesssim \sqrt{c_{0}} \cdot n(\boldsymbol{g}(t), \lambda(t))^{2}
$$

and then we will check that

$$
\left|\frac{\mathrm{d}}{\mathrm{d} t}\left\langle\mathrm{D} E\left(\boldsymbol{u}^{*}(t)\right), \boldsymbol{g}\right\rangle\right| \lesssim_{R} n(\boldsymbol{g}(t), \lambda(t))^{2} .
$$

Clearly, integrating (2.33) and using (2.32), we obtain the conclusion for $t_{0}$ sufficiently close to $T_{+}$. Note that the constant in (2.33) is allowed to depend on $R$ (because $T_{+}-t_{0}$ can also be chosen depending on $R$ ).

In order to prove (2.32), we begin by verifying that

$$
\left|\left\langle\mathrm{D} E\left(\boldsymbol{V}(\lambda)+\boldsymbol{u}^{*}\right), \boldsymbol{g}\right\rangle-\langle\mathrm{D} E(\boldsymbol{V}(\lambda)), \boldsymbol{g}\rangle-\left\langle\mathrm{D} E\left(\boldsymbol{u}^{*}\right), \boldsymbol{g}\right\rangle\right| \ll n(\boldsymbol{g}, \lambda)^{2} .
$$

This is equivalent to

$$
\int\left|f\left(V(\lambda)+u^{*}\right)-f(V(\lambda))-f\left(u^{*}\right)\right| \cdot|g| \mathrm{d} x \ll n(\boldsymbol{g}, \lambda)^{2} .
$$

By Hölder and Sobolev inequalities, it suffices to show that

$$
\left\|f\left(V(\lambda)+u^{*}\right)-f(V(\lambda))-f\left(u^{*}\right)\right\|_{L^{\frac{2 N}{N+2}}} \ll \lambda^{\frac{N-2}{4}} .
$$

Using (2.22) we obtain easily that the left hand side is $\lesssim \lambda^{\frac{N-2}{2}}$.

Recall that $R^{-N+2}=c_{0} c^{*}$, hence (2.5) gives $\left\|W_{\lambda}-V(\lambda)\right\|_{\dot{H}^{1}} \lesssim \sqrt{c_{0} c^{*}}$. Using $\Delta W_{\lambda}+f\left(W_{\lambda}\right)=0$ and the pointwise bound $\left|f\left(W_{\lambda}\right)-f(V(\lambda))\right| \lesssim f^{\prime}\left(W_{\lambda}\right) \cdot\left|W_{\lambda}-V(\lambda)\right|$ one gets

$$
\|\Delta V(\lambda)+f(V(\lambda))\|_{\dot{H}^{-1}} \lesssim\left\|\Delta\left(W_{\lambda}-V(\lambda)\right)\right\|_{\dot{H}^{-1}}+\left\|f\left(W_{\lambda}\right)-f(V(\lambda))\right\|_{L^{\frac{2 N}{N+2}}} \lesssim \sqrt{c_{0} c^{*}}
$$

hence

$$
|\langle\mathrm{D} E(\boldsymbol{V}(\lambda)), \boldsymbol{g}\rangle| \lesssim \sqrt{c_{0}} \cdot n(\boldsymbol{g}, \lambda)^{2} .
$$

Estimate (2.32) follows from (2.34) and (2.35). Notice that until now the assumption $\boldsymbol{u}_{0}^{*} \in X^{2} \times H^{2}$ has not been used, thus (2.32) holds also in the case $\boldsymbol{u}_{0}^{*} \in X^{s} \times H^{s}, s>\frac{N-2}{2}$.

We move on to the proof of (2.33). Until the end of this proof all the constants are allowed to depend on $R$. From (2.17) we get

$$
\frac{\mathrm{d}}{\mathrm{d} t}\left\langle\mathrm{D} E\left(\boldsymbol{u}^{*}\right), \boldsymbol{g}\right\rangle=\left\langle\mathrm{D}^{2} E\left(\boldsymbol{u}^{*}\right) \partial_{t} \boldsymbol{u}^{*}, \boldsymbol{g}\right\rangle+\left\langle\mathrm{D} E\left(\boldsymbol{u}^{*}\right), J \circ\left(\mathrm{D} E\left(\boldsymbol{V}(\lambda)+\boldsymbol{u}^{*}+\boldsymbol{g}\right)-\mathrm{D} E\left(\boldsymbol{u}^{*}\right)\right)-\lambda^{\prime} \partial_{\lambda} \boldsymbol{V}(\lambda)\right\rangle .
$$

Notice that

$$
\left\langle\mathrm{D}^{2} E\left(\boldsymbol{u}^{*}\right) \partial_{t} \boldsymbol{u}^{*}, \boldsymbol{g}\right\rangle=-\left\langle\mathrm{D} E\left(\boldsymbol{u}^{*}\right), J \circ \mathrm{D}^{2} E\left(\boldsymbol{u}^{*}\right) \boldsymbol{g}\right\rangle,
$$

hence it suffices to verify that

$$
\left|\left\langle\mathrm{D} E\left(\boldsymbol{u}^{*}\right), J \circ\left(\mathrm{D} E\left(\boldsymbol{V}(\lambda)+\boldsymbol{u}^{*}+\boldsymbol{g}\right)-\mathrm{D} E\left(\boldsymbol{u}^{*}\right)-\mathrm{D}^{2} E\left(\boldsymbol{u}^{*}\right) \boldsymbol{g}\right)-\lambda^{\prime} \partial_{\lambda} \boldsymbol{V}(\lambda)\right\rangle\right| \lesssim n(\boldsymbol{g}, \lambda)^{2} .
$$

Considering separately the first and the second component, cf. (2.18), we obtain that it is sufficient to verify the following bounds:

$$
\begin{aligned}
\left|\left\langle\Delta u^{*}+f\left(u^{*}\right), \lambda^{\prime} \partial_{\lambda} V(\lambda)\right\rangle\right| & \lesssim n(\boldsymbol{g}, \lambda)^{2}, \\
\left|\left\langle\dot{u}^{*}, f\left(V(\lambda)+u^{*}+g\right)-f\left(V_{\lambda}\right)-f\left(u^{*}\right)-f^{\prime}\left(u^{*}\right) g\right\rangle\right| & \lesssim n(\boldsymbol{g}, \lambda)^{2}, \\
\left|\left\langle\dot{u}^{*}, \Delta V(\lambda)+f(V(\lambda))\right\rangle\right| & \lesssim n(\boldsymbol{g}, \lambda)^{2} .
\end{aligned}
$$


We know from Appendix $\mathrm{A}$ that $u^{*}(t)$ is bounded in $X^{2}$, hence $\Delta u^{*}+f\left(u^{*}\right)$ is bounded in $L^{\frac{2 N}{N-2}}$ by the Sobolev embedding. From (2.8) and Hölder inequality it follows that

$$
\left|\left\langle\Delta u^{*}+f\left(u^{*}\right), \partial_{\lambda} V(\lambda)\right\rangle\right| \lesssim \lambda^{\frac{N-2}{4}},
$$

and (2.36) follows from (2.15).

Since $\dot{u}^{*}(t)$ is bounded in $L^{\frac{2 N}{N-2}}$, in order to prove (2.37) it suffices (by Hölder) to check that

$$
\left\|f\left(V(\lambda)+u^{*}+g\right)-f(V(\lambda))-f\left(u^{*}\right)-f^{\prime}\left(V(\lambda)+u^{*}\right) g\right\|_{L^{\frac{2 N}{N+2}}} \lesssim n(\boldsymbol{g}, \lambda)^{2}
$$

and

$$
\left\|\dot{u}^{*} \cdot\left(f^{\prime}\left(V(\lambda)+u^{*}\right)-f^{\prime}\left(u^{*}\right)\right)\right\|_{L^{\frac{2 N}{N+2}}} \lesssim \lambda^{\frac{N-2}{4}} .
$$

We first prove (2.40). For $|x| \geq R \sqrt{\lambda}$ the integrand equals 0 , and in the region $|x| \leq R \sqrt{\lambda}$ there holds $\left|f^{\prime}(V(\lambda))\right|+\left|f^{\prime}\left(u^{*}\right)\right| \lesssim f^{\prime}\left(W_{\lambda}\right)$.

- For $N=3 \dot{u}^{*} \in H^{2} \subset L^{\infty}$ and $\left\|f^{\prime}\left(W_{\lambda}\right)\right\|_{L^{\frac{6}{5}}} \lesssim \lambda^{\frac{1}{2}}$.

- For $N=4 \dot{u}^{*} \in H^{2} \subset L^{12}$ and $\left\|f^{\prime}\left(W_{\lambda}\right)\right\|_{L^{\frac{3}{2}}} \lesssim \lambda^{\frac{2}{3}}$.

- For $N=5 \dot{u}^{*} \in H^{2} \subset L^{10}$ and $\left\|f^{\prime}\left(W_{\lambda}\right)\right\|_{L^{\frac{5}{3}}} \lesssim \lambda$.

In all three cases (2.40) follows from Hölder inequality.

By a pointwise bound we have

$$
\left\|f\left(V(\lambda)+u^{*}\right)-f(V(\lambda))-f\left(u^{*}\right)\right\|_{L^{\frac{2 N}{N+2}}} \lesssim\left\|u^{*} \cdot f^{\prime}(V(\lambda))\right\|_{L^{\frac{2 N}{N+2}}}+\left\|f^{\prime}\left(u^{*}\right) \cdot V(\lambda)\right\|_{L^{\frac{2 N}{N+2}}} \cdot
$$

It is easy to check that

$$
\left\|f^{\prime}(V(\lambda))\right\|_{L^{\frac{2 N}{N+2}}} \leq\left\|f^{\prime}\left(W_{\lambda}\right)\right\|_{L^{\frac{2 N}{N+2}}} \lesssim \lambda^{\frac{N-2}{2}} .
$$

Together with (2.8) this yields

$$
\left\|f\left(V(\lambda)+u^{*}\right)-f(V(\lambda))-f\left(u^{*}\right)\right\|_{L^{\frac{2 N}{N+2}}} \lesssim \lambda^{\frac{N-2}{2}},
$$

and (2.39) follows from (2.24) and the Hölder inequality.

In order to prove (2.38), we write:

$$
\left|\left\langle\dot{u}^{*}, \Delta V(\lambda)+f(V(\lambda))\right\rangle\right| \leq\left|\left\langle\dot{u}^{*}, \Delta\left(V(\lambda)-W_{\lambda}\right)\right\rangle\right|+\mid\left\langle\dot{u}^{*}, f\left(V(\lambda)-f\left(W_{\lambda}\right)\right\rangle\right| .
$$

Consider the first term of (2.41). Integrating twice by parts we find

$$
\begin{aligned}
\int \dot{u}^{*} \cdot \Delta\left(V(\lambda)-W_{\lambda}\right) \mathrm{d} x & =\int_{|x| \geq R \sqrt{\lambda}} \nabla \dot{u}^{*} \cdot \nabla\left(W_{\lambda}\right) \mathrm{d} x \\
& =\int_{S(0, R \sqrt{\lambda})} \dot{u}^{*} \cdot \partial_{r}\left(W_{\lambda}\right) \mathrm{d} \sigma-\int_{|x| \geq R \sqrt{\lambda}} \dot{u}^{*} \cdot \Delta\left(W_{\lambda}\right) \mathrm{d} x .
\end{aligned}
$$

As for the first term, recall that $\left|\partial_{r}\left(W_{\lambda}(R \sqrt{\lambda})\right)\right| \lesssim \lambda^{\frac{N-2}{2}}$, so it suffices to notice that by the Trace Theorem $\int\left|\dot{u}^{*}\right| \mathrm{d} \sigma \ll 1$ for $\lambda \ll 1$. In order to bound the second term, we compute

$$
\left\|f\left(W_{\lambda}\right)\right\|_{L^{\frac{2 N}{N+2}}(|x| \geq R \sqrt{\lambda})}=\|f(W)\|_{L^{\frac{2 N}{N+2}}(|x| \geq R / \sqrt{\lambda})} \sim \lambda^{\frac{N+2}{4}} \ll \lambda^{\frac{N-2}{2}},
$$

and use Hölder.

Consider the second term of (2.41). From (2.6) we have $\left|f(V(\lambda))-f\left(W_{\lambda}\right)\right| \lesssim f^{\prime}\left(W_{\lambda}\right)$, hence:

$$
\left|\int \dot{u}^{*} \cdot\left(f(V(\lambda))-f\left(W_{\lambda}\right)\right) \mathrm{d} x\right| \lesssim \int\left|\dot{u}^{*}\right| \cdot f^{\prime}\left(W_{\lambda}\right) \mathrm{d} x,
$$

and the required bound follows from Hölder and the fact that $\left\|f^{\prime}\left(W_{\lambda}\right)\right\|_{L^{\frac{2 N}{N+2}}} \lesssim \lambda^{\frac{N-2}{2}}$. 
Lemma 2.9. Suppose that $\boldsymbol{u}_{0}^{*} \in X^{s} \times H^{s}, s>\frac{N-2}{2}$ and $s \geq 1$. There exists a decomposition

$$
\left\langle\mathrm{D} E\left(\boldsymbol{V}(\lambda(t))+\boldsymbol{u}^{*}(t)\right), \boldsymbol{g}(t)\right\rangle=b_{1}(t)+b_{2}(t)
$$

such that for $t \in\left[t_{0}, T_{+}\right)$there holds:

$$
\begin{aligned}
\left|b_{1}^{\prime}(t)\right| \ll \lambda(t)^{\frac{N-4}{2}}\|\boldsymbol{g}\|_{\mathcal{E}}, \\
\left|b_{2}(t)\right| \lesssim \sqrt{c_{0}} \cdot \sup _{t \leq \tau<T_{+}} n(\boldsymbol{g}(\tau), \lambda(\tau))^{2} .
\end{aligned}
$$

Proof. We take

$$
\begin{aligned}
& b_{1}(t):=\left\langle\mathrm{D} E\left(\boldsymbol{u}^{*}(t)\right), \boldsymbol{g}(t)\right\rangle, \\
& b_{2}(t):=\left\langle\mathrm{D} E\left(\boldsymbol{V}(\lambda(t))+\boldsymbol{u}^{*}(t)\right)-\mathrm{D} E\left(\boldsymbol{u}^{*}(t)\right), \boldsymbol{g}(t)\right\rangle .
\end{aligned}
$$

Estimate (2.43) is exactly (2.32).

Repeating the computation in the proof of Lemma 2.8, we see that we need to check inequalities (2.36), (2.37) and (2.38), with " $\lesssim n(\boldsymbol{g}, \lambda)^{2}$ " replaced by " $\ll \lambda^{\frac{N-4}{2}}\|\boldsymbol{g}\| "$.

We know that $\Delta u^{*}$ is bounded in $H^{s-1}$, hence from (2.9) we obtain $\left|\left\langle\Delta u^{*}, \partial_{\lambda} V(\lambda)\right\rangle\right| \ll \lambda^{\frac{N-4}{2}}$. Since $\left\|f\left(u^{*}\right)\right\|_{L^{\frac{2 N}{N-2}}}$ is bounded and $\frac{N-2}{4}>\frac{N-4}{2}$, from (2.8) we get $\left|\left\langle f\left(u^{*}\right), \partial_{\lambda} V(\lambda)\right\rangle\right| \ll \lambda^{\frac{N-4}{2}}$. Using (2.15), it follows that

$$
\left|\left\langle\Delta u^{*}+f\left(u^{*}\right), \lambda^{\prime} \partial_{\lambda} V(\lambda)\right\rangle\right| \ll \lambda^{\frac{N-4}{2}}\|\boldsymbol{g}\| .
$$

The proof of (2.37) applies almost without changes, but instead of (2.40) we need to check that $\left\|\dot{u}^{*} \cdot\left(f^{\prime}\left(V(\lambda)+u^{*}\right)-f^{\prime}\left(u^{*}\right)\right)\right\|_{L^{\frac{2 N}{N+2}}} \ll \lambda^{\frac{N-4}{2}}$, which will follow from

$$
\left\|\dot{u}^{*} \cdot f^{\prime}\left(W_{\lambda}\right)\right\|_{L^{\frac{2 N}{N+2}}} \ll \lambda^{\frac{N-4}{2}} .
$$

We check (2.44) separately for $N=3,4,5$. Recall that $\dot{u}^{*}$ is bounded in $H^{s}$. If $N=3$, then $\left\|\dot{u}^{*}\right\|_{L^{6}}$ and $\left\|f^{\prime}\left(W_{\lambda}\right)\right\|_{L^{\frac{3}{2}}}$ are bounded, hence (2.44) follows from Hölder. If $N=4$, then (by Sobolev) there exists $q>4$ such that $\left\|\dot{u}^{*}\right\|_{L^{q}}$ is bounded. It can be checked that for $1<p<2,\left\|f^{\prime}\left(W_{\lambda}\right)\right\|_{L^{p}} \ll 1$, hence (2.44) follows. If $N=5$, then there exists $q>5$ such that $\left\|\dot{u}^{*}\right\|_{L^{q}}$ is bounded. It can be checked that for $\frac{5}{4}<p<2,\left\|f^{\prime}\left(W_{\lambda}\right)\right\|_{L^{p}} \ll \sqrt{\lambda}$, hence (2.44) follows.

In the proof of (2.38) we have only used the boundedness of $\dot{u}^{*}$ in $H^{1}$, hence it remains valid and gives the bound

$$
\left|\dot{u}^{*}, \Delta V(\lambda)+f(V(\lambda))\right\rangle \mid \lesssim \lambda^{\frac{N-2}{2}} \ll \lambda^{\frac{N-2}{4}}
$$

Remark 2.10. It is not excluded that Lemma 2.8 holds under the assumption $\boldsymbol{u}_{0}^{*} \in X^{s} \times H^{s}$, $s>\frac{N-2}{2}$, but I was unable to prove it because of possible oscillations of $\lambda(t)$. Note also that Lemma 2.9 could be proved for less regular $\boldsymbol{u}_{0}^{*}$ if we had some control of $\boldsymbol{g}(t)$ in suitable (for example Strichartz) norms.

Lemma 2.8 implies that if $\boldsymbol{u}_{0}^{*} \in X^{2} \times H^{2}$, then Lemma 2.9 holds with $b_{1}(t)=0$.

For $t_{0} \leq t<T_{+}$we define

$$
\varphi(t):=C_{\mathrm{I}} c^{*} \lambda(t)^{\frac{N-2}{2}}-b_{1}(t)+2\left(a^{-}(t)^{2}+a^{+}(t)^{2}\right)
$$

$\left(C_{\mathrm{I}}\right.$ is a constant to be chosen shortly). From (2.28) we have

$$
\begin{aligned}
\varphi(t) & :=C_{\mathrm{I}} c^{*} \lambda(t)^{\frac{N-2}{2}}+\left(E\left(\boldsymbol{V}(\lambda)+\boldsymbol{u}^{*}\right)-E(\boldsymbol{W})-E\left(\boldsymbol{u}^{*}\right)\right) \\
& +\frac{1}{2}\left\langle\mathrm{D}^{2} E\left(\boldsymbol{V}(\lambda)+\boldsymbol{u}^{*}\right) \boldsymbol{g}, \boldsymbol{g}\right\rangle+2\left(a^{-}(t)^{2}+a^{+}(t)^{2}\right)+b_{2}(t)+O\left(\|\boldsymbol{g}\|_{\mathcal{E}}^{3}\right) .
\end{aligned}
$$


We will consider the maximal function:

$$
\varphi_{\mathrm{M}}(t):=\sup _{t \leq \tau<T_{+}} \varphi(\tau)
$$

Note that $\varphi_{\mathrm{M}}:\left[t_{0}, T_{+}\right) \rightarrow \mathbb{R}$ is decreasing, $\lim _{t \rightarrow T_{+}} \varphi_{\mathrm{M}}(t)=0$ and $0 \geq \varphi_{\mathrm{M}}^{\prime}(t) \geq \min \left(0, \varphi^{\prime}(t)\right)$ almost everywhere.

Corollary 2.11. Let $s>\frac{N-2}{2}$ and $s \geq 1$. For $t_{0} \leq t<T_{+}$there holds

$$
\varphi_{\mathrm{M}}(t) \sim \sup _{t \leq \tau<T_{+}} n(\boldsymbol{g}(\tau), \lambda(\tau))^{2} .
$$

Proof. Lemma 2.7 and (2.28) yield $\left|\left\langle\mathrm{D} E\left(\boldsymbol{V}(\lambda)+\boldsymbol{u}^{*}\right), \boldsymbol{g}\right\rangle\right| \lesssim n(\boldsymbol{g}, \lambda)^{2}$, hence from Lemma 2.9 we have

$$
\left|b_{1}(t)\right| \lesssim \sup _{t \leq \tau<T_{+}} n(\boldsymbol{g}(t), \lambda(t))^{2}
$$

Let $t \in\left[t_{0}, T_{+}\right)$and let $t_{1} \in\left[t, T_{+}\right)$be such that $\varphi_{\mathrm{M}}(t)=\varphi\left(t_{1}\right)$ (such $t_{1}$ exists by the definition of $\varphi_{\mathrm{M}}$ ). Using (2.47) we obtain

$$
\varphi_{\mathrm{M}}(t)=\varphi\left(t_{1}\right) \lesssim \sup _{t_{1} \leq \tau<T_{+}} n(\boldsymbol{g}(\tau), \lambda(\tau))^{2} \leq \sup _{t \leq \tau<T_{+}} n(\boldsymbol{g}(\tau), \lambda(\tau))^{2} .
$$

Now let $t_{2} \in\left[t, T_{+}\right)$be such that $\sup _{t \leq \tau<T_{+}} n(\boldsymbol{g}(\tau), \lambda(\tau))^{2}=n\left(\boldsymbol{g}\left(t_{2}\right), \lambda\left(t_{2}\right)\right)^{2}$. From Lemma 2.2 and the fact that $\left\|\boldsymbol{V}(\lambda)+\boldsymbol{u}^{*}-\boldsymbol{W}_{\lambda}\right\|_{\mathcal{E}}$ is small we obtain

$$
\frac{1}{2}\left\langle\mathrm{D}^{2} E\left(\boldsymbol{V}\left(\lambda\left(t_{2}\right)\right)+\boldsymbol{u}^{*}\left(t_{2}\right)\right) \boldsymbol{g}\left(t_{2}\right), \boldsymbol{g}\left(t_{2}\right)\right\rangle+2\left(a^{-}\left(t_{2}\right)^{2}+a^{+}\left(t_{2}\right)^{2}\right) \gtrsim\left\|\boldsymbol{g}\left(t_{2}\right)\right\|_{\mathcal{E}}^{2}
$$

From Lemma 2.7, if we choose $C_{\mathrm{I}}$ large enough, then $C_{\mathrm{I}} c^{*} \lambda^{\frac{N-2}{2}}+E\left(\boldsymbol{V}(\lambda)+\boldsymbol{u}^{*}\right)-E(\boldsymbol{W})-E\left(\boldsymbol{u}^{*}\right) \gtrsim$ $c^{*} \lambda^{\frac{N-2}{2}}$, hence (2.46) and (2.48) yield

$$
\varphi\left(t_{2}\right)-b_{2}\left(t_{2}\right) \gtrsim n\left(\boldsymbol{g}\left(t_{2}\right), \lambda\left(t_{2}\right)\right)^{2}
$$

From Lemma 2.9 we have $\left|b_{2}\left(t_{2}\right)\right| \leq \sqrt{c_{0}} \cdot \sup _{t_{2} \leq \tau<T_{+}} n(\boldsymbol{g}(\tau), \lambda(\tau))^{2}=\sqrt{c_{0}} \cdot n\left(\boldsymbol{g}\left(t_{2}\right), \lambda\left(t_{2}\right)\right)^{2}$, hence we obtain

$$
\varphi_{\mathrm{M}}(t) \geq \varphi\left(t_{2}\right) \gtrsim n\left(\boldsymbol{g}\left(t_{2}\right), \lambda\left(t_{2}\right)\right)^{2}=\sup _{t \leq \tau<T_{+}} n(\boldsymbol{g}(\tau), \lambda(\tau))^{2},
$$

provided that $c_{0}$ is small enough.

\subsection{Differential inequalities and conclusion.}

Lemma 2.12. There exists a constant $C_{a}$ such that for $T_{+}-t$ small enough there holds

$$
\left|a^{+}(t)\right| \leq C_{a} \cdot \sup _{t \leq \tau<T_{+}} n(\boldsymbol{g}(\tau), \lambda(\tau))^{2}, \quad\left|a^{-}(t)\right| \leq C_{a} \cdot \sup _{t \leq \tau<T_{+}} n(\boldsymbol{g}(\tau), \lambda(\tau))^{2} .
$$

Proof. It follows from (2.19) that there exists $C_{1}>0$ such that

$$
\left|a^{+}(t)\right| \geq C_{1} \cdot n(\boldsymbol{g}(t), \lambda(t))^{2} \Rightarrow \frac{\mathrm{d}}{\mathrm{d} t}\left|a^{+}(t)\right| \geq \frac{\nu}{2 \lambda(t)}\left|a^{+}(t)\right| .
$$

Suppose that

$$
\left|a^{+}(t)\right| \geq 2 C_{1} \cdot \sup _{t \leq \tau<T_{+}} n(\boldsymbol{g}(\tau), \lambda(\tau))^{2}
$$

and suppose that $t_{1} \in\left[t, T_{+}\right)$is the smallest time such that

$$
\left|a^{+}\left(t_{1}\right)\right| \leq C_{1} \cdot \sup _{t_{1} \leq \tau<T_{+}} n(\boldsymbol{g}(\tau), \lambda(\tau))^{2} .
$$

Clearly $t_{1}>t$. The function on the right hand side is decreasing with respect to $t_{1}$, hence $\frac{\mathrm{d}}{\mathrm{d} t}\left|a^{+}(t)\right|_{t=t_{1}} \leq 0$. This contradicts (2.49), hence for all $t^{\prime} \in\left[t, T_{+}\right)$we have

$$
\left|a^{+}\left(t^{\prime}\right)\right| \geq C_{1} \cdot n\left(\boldsymbol{g}\left(t^{\prime}\right), \lambda\left(t^{\prime}\right)\right)^{2} .
$$


Observe that

$$
\int_{t}^{T_{+}} \frac{1}{\lambda(\tau)} \mathrm{d} \tau \gtrsim \int_{t}^{T_{+}} \frac{\left|\lambda^{\prime}(\tau)\right|}{\lambda(\tau)} \mathrm{d} \tau=+\infty .
$$

From (2.50), (2.49) and (2.51) we obtain $\left|a^{+}(t)\right| \rightarrow+\infty$ as $t \rightarrow T_{+}$, a contradiction.

We will now consider $a^{-}(t)$, which is less straightforward. It follows from (2.19) that there exists $C_{2}>0$ such that

$$
\left|a^{-}(t)\right| \geq C_{2} \cdot n(\boldsymbol{g}(t), \lambda(t))^{2} \Rightarrow \frac{\mathrm{d}}{\mathrm{d} t}\left|a^{-}(t)\right| \leq-\frac{\nu}{2 \lambda(t)}\left|a^{-}(t)\right| .
$$

From Corollary 2.11 we obtain existence of a constant $C_{3}>0$ such that

$$
\left|a^{-}(t)\right| \geq C_{3} \cdot \varphi_{\mathrm{M}}(t) \quad \Rightarrow \quad\left|a^{-}(t)\right| \geq C_{2} \cdot n(\boldsymbol{g}(t), \lambda(t))^{2}
$$

and a constant $C_{4}>0$ such that

$$
\left|a^{-}(t)\right| \geq C_{4} \cdot \sup _{t \leq \tau<T_{+}} n(\boldsymbol{g}(t), \lambda(t))^{2} \quad \Rightarrow \quad\left|a^{-}(t)\right| \geq 2 C_{3} \cdot \varphi_{\mathrm{M}}(t) .
$$

Suppose that $t \in\left[t_{0}, T_{+}\right)$is such that

$$
\left|a^{-}(t)\right| \geq C_{4} \cdot \sup _{t \leq \tau<T_{+}} n(\boldsymbol{g}(\tau), \lambda(\tau))^{2}
$$

and let $t_{1} \in\left[t_{0}, t\right]$ be the smallest time such that for $t^{\prime} \in\left[t_{1}, t\right]$ there holds

$$
\left|a^{-}\left(t^{\prime}\right)\right| \geq C_{3} \cdot \varphi_{\mathrm{M}}\left(t^{\prime}\right)
$$

Of course $t_{1}<t$. Suppose that $t_{1}>t_{0}$. This implies

$$
-\frac{C_{2} \nu}{\lambda\left(t_{1}\right)} n\left(\boldsymbol{g}\left(t_{1}\right), \lambda\left(t_{1}\right)\right)^{2} \geq-\frac{\nu}{2 \lambda\left(t_{1}\right)}\left|a^{-}\left(t_{1}\right)\right| \geq \frac{\mathrm{d}}{\mathrm{d} t}\left|a^{-}(t)\right|_{t=t_{1}} \geq C_{3} \cdot \varphi_{\mathrm{M}}^{\prime}\left(t_{1}\right)
$$

(we use respectively (2.53), (2.52) and the definition of $t_{1}$ ).

However, $\left|\varphi_{\mathrm{M}}^{\prime}\left(t_{1}\right)\right| \leq\left|\varphi^{\prime}\left(t_{1}\right)\right| \ll \frac{1}{\lambda\left(t_{1}\right)} n\left(\boldsymbol{g}\left(t_{1}\right), \lambda\left(t_{1}\right)\right)^{2}$, as is easily seen from (2.45). The contradiction shows that $t_{1}=t_{0}$, hence (2.55) holds for $t^{\prime} \in\left[t_{0}, t\right]$. This means that if there exist times $t$ arbitrarily close to $T_{+}$such that (2.54) holds, then (2.55) is true for $t^{\prime} \in\left[t_{0}, T_{+}\right)$. From (2.52) and (2.53) we deduce that for $t \in\left[t_{0}, T_{+}\right)$there holds

$$
\left|a^{-}(t)\right| \leq\left|a^{-}\left(t_{0}\right)\right| \cdot \exp \left(-\int_{t_{0}}^{t} \frac{\nu \mathrm{d} t}{2 \lambda(t)}\right) .
$$

By (2.53) and (2.15), this implies

$$
\left|\lambda^{\prime}(t)\right| \lesssim \exp \left(-\int_{t_{0}}^{t} \frac{\nu \mathrm{d} t}{4 \lambda(t)}\right) .
$$

Dividing both sides by $\lambda(t)$ and integrating we get a contradiction.

We have proved the lemma with $C_{a}:=\max \left(2 C_{1}, C_{4}\right)$.

By modifying $t_{0}$ we can assume that Lemma 2.12 holds for $t \in\left[t_{0}, T_{+}\right)$.

Proof of Theorem 1. We define

$$
\widetilde{\varphi}(t):=C_{\mathrm{I}} c^{*} \lambda(t)^{\frac{N-2}{2}}-b_{1}(t), \quad \widetilde{\varphi}_{\mathrm{M}}(t):=\sup _{t \leq \tau<T_{+}} \widetilde{\varphi}(\tau) .
$$

From Lemma 2.12 and Corrolary 2.11, it is clear that

$$
\widetilde{\varphi}_{\mathrm{M}}(t) \sim \sup _{t \leq \tau<T_{+}} n(\boldsymbol{g}(\tau), \lambda(\tau))^{2} .
$$


We will consider first the case $N \in\{4,5\}$. Using (2.42) and (2.56) we obtain the following differential inequality for $t \in\left[t_{0}, T_{+}\right)$:

$$
\left|\widetilde{\varphi}_{\mathrm{M}}^{\prime}(t)\right| \leq\left|\widetilde{\varphi}^{\prime}(t)\right| \lesssim c^{*} \lambda(t)^{\frac{N-4}{2}}\|\boldsymbol{g}(t)\|_{\mathcal{E}} \lesssim\left(c^{*}\right)^{\frac{2}{N-2}} \widetilde{\varphi}_{\mathrm{M}}(t)^{\frac{3 N-10}{2(N-2)}}
$$

Integrating this inequality we find

$$
\widetilde{\varphi}_{\mathrm{M}}(t) \lesssim\left(c^{*}\right)^{\frac{4}{6-N}}\left(T_{+}-t\right)^{\frac{2(N-2)}{6-N}} .
$$

To finish the proof, recall that $c^{*} \lambda(t)^{\frac{N-2}{2}} \lesssim \widetilde{\varphi}_{\mathrm{M}}(t)$ by Corollary 2.11

Consider now the case $N=3$. The problem is that $N-4<0$, hence we cannot write $\left(c^{*} \lambda(t)\right)^{\frac{N-4}{2}} \lesssim \widetilde{\varphi}_{\mathrm{M}}^{\frac{N-4}{2(N-2)}}$, as we did in the previous proof. Instead, we just have

$$
\left|\widetilde{\varphi}_{\mathrm{M}}^{\prime}(t)\right| \lesssim c^{*} \lambda(t)^{-\frac{1}{2}} \cdot \sqrt{\widetilde{\varphi}_{\mathrm{M}}(t)}
$$

Integrating between $t$ and $T_{+}$we obtain

$$
\sqrt[4]{\lambda(t)} \lesssim \sqrt{c^{*}} \int_{t}^{T_{+}} \frac{\mathrm{d} \tau}{\sqrt{\lambda(\tau)}}
$$

This is again a differential inequality. It yields (1.6).

Remark 2.13. In the case $N=3$ and $\boldsymbol{u}^{*} \in X^{2} \times H^{2}$, we can prove (1.4) for continuous time, not only for a sequence. Indeed, in this case one can take $b_{1}(t)=0$ (see Remark 2.10), hence $\widetilde{\varphi}_{\mathrm{M}}(t)=C_{\mathrm{I}} c^{*} \sqrt{\lambda(t)}$. If $t \in\left[t_{0}, T_{+}\right)$is such that $\lambda(t)<\sup _{t \leq \tau<T_{+}} \lambda(\tau)$, then obviously $\widetilde{\varphi}_{\mathrm{M}}^{\prime}(t)=0$. If $\lambda(t)=\sup _{t \leq \tau<T_{+}} \lambda(\tau)$, then $c^{*} \sqrt{\lambda(t)} \sim \widetilde{\varphi}_{\mathrm{M}}(t)$, hence the proof of (2.57) applies. The end of the proof is the same as in the case $N \in\{4,5\}$.

Proof of Theorem Q Let $t \in\left[t_{0}, T_{+}\right)$be such that $n(\boldsymbol{g}(t), \lambda(t))=\sup _{t \leq \tau<T_{+}} n(\boldsymbol{g}(\tau), \lambda(\tau))$. From (2.29) and Lemma 2.2 we get

$$
\left(E\left(\boldsymbol{V}(\lambda)+\boldsymbol{u}^{*}\right)-E(\boldsymbol{W})-E\left(\boldsymbol{u}^{*}\right)\right)+\frac{1}{2}\left\langle\mathrm{D}^{2} E\left(\boldsymbol{V}(\lambda)+\boldsymbol{u}^{*}\right) \boldsymbol{g}, \boldsymbol{g}\right\rangle+2\left(\left(a^{-}\right)^{2}+\left(a^{+}\right)^{2}\right) \gtrsim n(\boldsymbol{g}, \lambda)^{2} .
$$

But due to Lemma 2.12, the last term on the right hand side can be omitted. This is in contradiction with (2.28) and Lemma 2.8 .

\section{Appendix A. CAuchy theory in higher Regularity}

In this section we prove some facts about propagation of regularity for (NLW), which are applied to $\boldsymbol{u}^{*}(t)$ in the main text. As in [13, Appendix B], the proofs rely on the classical energy estimates:

Proposition. Let $s \geq 0, t_{0} \in\left[T_{1}, T_{2}\right], g \in L^{1}\left(I, H^{s}\right)$ and $\boldsymbol{u}_{0} \in X^{s} \times H^{s}$. Then the solution of the linear wave equation $\left(\partial_{t t}-\Delta\right) u=g$ with initial data $\boldsymbol{u}\left(t_{0}\right)=\boldsymbol{u}_{0}$ satifies

$$
\|\boldsymbol{u}(t)\|_{X^{s} \times H^{s}} \leq\left\|\boldsymbol{u}_{0}\right\|_{X^{s} \times H^{s}}+\left|\int_{t_{0}}^{t}\|g(\tau)\|_{H^{s}} \mathrm{~d} \tau\right|, \quad \forall t \in\left[T_{1}, T_{2}\right] .
$$

Proposition A.1. Let $N \in\{3,4\}, s>\frac{N-2}{2}$ and $\boldsymbol{u}_{0} \in X^{s} \times H^{s}$. There exist $t_{1}<t_{0}<t_{2}$ such that the solution $\boldsymbol{u}(t)$ of (NLW) satisfies

$$
\boldsymbol{u} \in C\left(\left[t_{1}, t_{2}\right], X^{s} \times H^{s}\right) .
$$

Proof. This is a standard application of the energy estimates and the Fixed Point Theorem, using the fact that $f(u)$ is a monomial and $X^{s} \hookrightarrow L^{\infty}$. We skip the details. 
In the rest of this section we consider (NLW) in dimension $N=5$. In this case the nonlinearity $f(u)=|u|^{\frac{4}{3}} u$ is not smooth. We will use the following regularization:

$$
f_{n}(u):=(1-\chi(n u)) f(u), \quad n \in\{1,2,3, \ldots\},
$$

where

$$
\chi \in C^{\infty}, \quad \chi(-u)=\chi(u), \quad \chi(u)=1 \text { for } u \in[-1,1], \quad \operatorname{supp} \chi \subset[-2,2] .
$$

In the proof of the next result we will use the Fractional Leibniz Rule and the Fractional Chain Rule in the form given in [3, Propositions 3.1, 3.3]:

\section{Proposition A.2.}

- If $\Psi \in C^{1}, 0<\alpha<1$ and $1<p, p_{1}, p_{2}$ are such that $\frac{1}{p}=\frac{1}{p_{1}}+\frac{1}{p_{2}}$, then

$$
\left\||\nabla|^{\alpha} \Psi(u)\right\|_{L^{p}} \lesssim\left\|\Psi^{\prime}(u)\right\|_{L^{p_{1}}} \cdot\left\||\nabla|^{\alpha} u\right\|_{L^{p_{2}}} .
$$

- If $0<\alpha<1$ and $1<p, p_{1}, p_{2}, \widetilde{p}_{1}, \widetilde{p}_{2}$ are such that $\frac{1}{p}=\frac{1}{p_{1}}+\frac{1}{p_{2}}=\frac{1}{\widetilde{p}_{1}}+\frac{1}{\widetilde{p}_{2}}$, then

$$
\left\||\nabla|^{\alpha}(u v)\right\|_{L^{p}} \lesssim\left\||\nabla|^{\alpha} u\right\|_{L^{p_{1}}} \cdot\|v\|_{L^{p_{2}}}+\|u\|_{L^{\widetilde{p}_{1}}} \cdot\left\||\nabla|^{\alpha} v\right\|_{L^{\widetilde{p}_{2}}} \cdot
$$

Remark A.3. In 3, the Leibniz Rule and the Chain Rule are proved in the case of one space dimension, and necessary changes in order to carry out a proof in arbitrary dimension are indicated. In the present paper we use this result in dimension 5, but only for radial functions, and it can be verified that the Leibniz Rule and the Chain Rule for radial functions is a consequence of the one-dimensional result.

Lemma A.4. Let $N=5$ and $1 \leq s \leq 2$. The following estimates hold (with constants which may depend on $s$ ):

$$
\begin{aligned}
\left\|f(u)-f_{n}(u)\right\|_{H^{1}} & \leq c_{n}\left(1+f\left(\|u\|_{X^{1}}\right)\right), \quad \text { with } c_{n} \rightarrow 0 \text { as } n \rightarrow+\infty \\
\|f(u)-f(v)\|_{H^{1}} & \lesssim\|u-v\|_{X^{1}} \cdot\left(f^{\prime}\left(\|u\|_{X^{1}}\right)+f^{\prime}\left(\|v\|_{X^{1}}\right)\right) \\
\left\|f_{n}(u)-f_{n}(v)\right\|_{H^{1}} & \lesssim\|u-v\|_{X^{1}} \cdot\left(f^{\prime}\left(\|u\|_{X^{1}}\right)+f^{\prime}\left(\|v\|_{X^{1}}\right)\right) \\
\|f(u)\|_{H^{s}} & \lesssim f\left(\|u\|_{X^{s}}\right) \\
\left\|f_{n}(u)\right\|_{H^{s}} & \lesssim f\left(\|u\|_{X^{s}}\right), \\
\left\|f_{n}(u)-f_{n}(v)\right\|_{H^{s}} & \leq C_{n}\|u-v\|_{X^{s}} \cdot\left(1+f^{\prime}\left(\|u\|_{X^{s}}\right)+f^{\prime}\left(\|v\|_{X^{s}}\right)\right), \quad C_{n}>0
\end{aligned}
$$

where the sign $\lesssim$ means that the constant is independent of $n$.

Proof. A simple computation shows that

$$
\begin{gathered}
\left|f_{n}(u)\right| \leq|f(u)|, \quad\left|f_{n}^{\prime}(u)\right| \lesssim\left|f^{\prime}(u)\right|, \quad\left|f_{n}^{\prime \prime}(u)\right| \lesssim\left|f^{\prime \prime}(u)\right|, \\
f_{n} \rightarrow f \quad \text { in } C^{2}(\mathbb{R}), \\
\left|f_{n}^{\prime \prime \prime}(u)\right| \lesssim n^{\frac{2}{3}}
\end{gathered}
$$

We have

$$
\left\|\nabla\left(f(u)-f_{n}(u)\right)\right\|_{L^{2}}=\left\|\left(f^{\prime}(u)-f_{n}^{\prime}(u)\right) \nabla u\right\|_{L^{2}} \leq\left\|f^{\prime}-f_{n}^{\prime}\right\|_{L^{\infty}} \cdot\|u\|_{H^{1}},
$$

which is acceptable due to A.7).

In order to bound $\left\|f(u)-f_{n}(u)\right\|_{L^{2}}$, we interpolate between $\left\|f-f_{n}\right\|_{L^{\infty}}$ and

$$
\left\|f(u)-f_{n}(u)\right\|_{L^{\frac{10}{7}}} \lesssim f\left(\|u\|_{L^{\frac{10}{3}}}\right) \lesssim f\left(\|u\|_{H^{1}}\right) .
$$

This proves (A.1).

Estimate (A.2) is a part of [13, Lemma B.3] and the proof of (A.3) is analogous. 
From the Sobolev inequality we get $\left\|f_{n}(u)\right\|_{L^{2}} \leq\|f(u)\|_{L^{2}} \leq f\left(\|u\|_{L^{\frac{14}{3}}}\right) \lesssim f\left(\|u\|_{X^{s}}\right)$, hence in order to prove (A.4) and (A.5) it suffices to check that

$$
\left\||\nabla|^{s}(f(u))\right\|_{L^{2}} \lesssim f\left(\|u\|_{X^{s}}\right), \quad\left\||\nabla|^{s}\left(f_{n}(u)\right)\right\|_{L^{2}} \lesssim f\left(\|u\|_{X^{s}}\right)
$$

For $s \in\{1,2\}$ this is an easy algebraic computation which we will skip. For $1<s<2$ we use Proposition A.2.

$$
\begin{aligned}
\left\||\nabla|^{s}(f(u))\right\|_{L^{2}} & =\left\||\nabla|^{s-1} \nabla(f(u))\right\|_{L^{2}}=\left\||\nabla|^{s-1}\left(f^{\prime}(u) \nabla u\right)\right\|_{L^{2}} \\
& \lesssim\left\||\nabla|^{s-1} \nabla u\right\|_{L^{\frac{10}{3}}} \cdot\left\|f^{\prime}(u)\right\|_{L^{5}}+\left\||\nabla|^{s-1}\left(f^{\prime}(u)\right)\right\|_{L^{5}} \cdot\|\nabla u\|_{L^{\frac{10}{3}}} \\
& \lesssim\left\||\nabla|^{s-1} \nabla u\right\|_{H^{1}} \cdot f^{\prime}\left(\|u\|_{L^{\frac{20}{3}}}\right)+\left\|f^{\prime \prime}(u)\right\|_{L^{10}} \cdot\left\|\left.\nabla\right|^{s-1} u\right\|_{L^{10}} \cdot\|\nabla u\|_{H^{1}} \\
& \lesssim f\left(\|u\|_{X^{s}}\right) .
\end{aligned}
$$

The second inequality in A.9 is proved analogously.

In order to prove (A.6) it suffices to check that

$$
\left\||\nabla|^{s}\left(f_{n}(u)-f_{n}(v)\right)\right\|_{L^{2}} \leq C_{n}\|u-v\|_{X^{s}} \cdot\left(1+f^{\prime}\left(\|u\|_{X^{s}}\right)+f^{\prime}\left(\|v\|_{X^{s}}\right)\right)
$$

(the estimate of $\left\|f_{n}(u)-f_{n}(v)\right\|_{L^{2}}$ is a part of (A.3) ). We write

$$
f_{n}(u)-f_{n}(v)=-(v-u) \int_{0}^{1} f_{n}^{\prime}((1-t) u+t v) \mathrm{d} t,
$$

hence by the triangle inequality

$$
\left\||\nabla|^{s}\left(f_{n}(u)-f_{n}(v)\right)\right\|_{L^{2}} \leq \int_{0}^{1}\left\||\nabla|^{s}\left((u-v) f_{n}^{\prime}((1-t) u+t v)\right)\right\|_{L^{2}} \mathrm{~d} t .
$$

We will estimate the integrand for fixed $t \in[0,1]$. We have

$$
\begin{aligned}
\left\||\nabla|^{s}\left((u-v) f_{n}^{\prime}((1-t) u+t v)\right)\right\|_{L^{2}} & =\left\||\nabla|^{s-1} \nabla\left((u-v) f_{n}^{\prime}((1-t) u+t v)\right)\right\|_{L^{2}} \\
& =\left\||\nabla|^{s-1}\left(\nabla(u-v) \cdot f_{n}^{\prime}((1-t) u+t v)\right)\right\|_{L^{2}} \\
& +\left\||\nabla|^{s-1}\left((u-v) \cdot((1-t) \nabla u+t \nabla v) \cdot f_{n}^{\prime \prime}((1-t) u+t v)\right)\right\|_{L^{2}} .
\end{aligned}
$$

The first term is estimated exactly as in (A.9), so we will only consider the second one. From the Leibniz Rule we obtain

$$
\begin{aligned}
& \left\||\nabla|^{s-1}\left((u-v) \cdot((1-t) \nabla u+t \nabla v) \cdot f_{n}^{\prime \prime}((1-t) u+t v)\right)\right\|_{L^{2}} \\
& \lesssim\left\||\nabla|^{s-1}(u-v)\right\|_{L^{10}} \cdot\|(1-t) u+t v\|_{L^{\frac{10}{3}}} \cdot\left\|f_{n}^{\prime \prime}((1-t) u+t v)\right\|_{L^{10}} \\
& +\|u-v\|_{L^{10}} \cdot\left\||\nabla|^{s-1}((1-t) \nabla u+t \nabla v)\right\|_{L^{\frac{10}{3}}} \cdot\left\|f_{n}^{\prime \prime}((1-t) u+t v)\right\|_{L^{10}} \\
& +\|u-v\|_{L^{p_{1}}} \cdot\|(1-t) \nabla u+t \nabla v\|_{L^{p_{2}}} \cdot\left\||\nabla|^{s-1} f_{n}^{\prime \prime}((1-t) u+t v)\right\|_{L^{p_{3}}},
\end{aligned}
$$

where the exponents $p_{1}, p_{2}, p_{3} \in(1,+\infty)$ are chosen such that $p_{1}>10, p_{2}>\frac{10}{3}, p_{3}<10, X^{s} \subset$ $L^{p_{1}} \cap W^{1, p_{2}}$ and $\frac{1}{2}=\frac{1}{p_{1}}+\frac{1}{p_{2}}+\frac{1}{p_{3}}$. Estimating the first two lines is straightforward and for the last line we use the Chain Rule together with (A.8).

Proposition A.5. Let $N=5,1 \leq s \leq 2$ and $\boldsymbol{u}_{0} \in X^{s} \times H^{s}$. There exist $t_{1}<t_{0}<t_{2}$ such that the solution $\boldsymbol{u}(t)$ of (NLW) satisfies

$$
\boldsymbol{u} \in C\left(\left[t_{1}, t_{2}\right], X^{s} \times H^{s}\right) .
$$

Proof. Using (A.4) for $s=1$ and (A.2) one obtains by a standard procedure that there exists a unique maximal solution

$$
\boldsymbol{u} \in C\left(\left[T_{1}, T_{2}\right], X^{1} \times H^{1}\right), \quad T_{1}<t_{0}<T_{2}
$$


and

$$
T_{1}>-\infty \Rightarrow \lim _{t \rightarrow T_{1}}\left\|\boldsymbol{u}_{n}\right\|_{X^{1} \times H^{1}}=+\infty, \quad T_{2}<+\infty \Rightarrow \lim _{t \rightarrow T_{2}}\left\|\boldsymbol{u}_{n}\right\|_{X^{1} \times H^{1}}=+\infty,
$$

see [13, Proposition B.2] for details.

Consider the regularized problem for $n \in\{1,2,3, \ldots\}$ :

$$
\left\{\begin{aligned}
\left(\partial_{t t}-\Delta\right) u_{n} & =f_{n}\left(u_{n}\right), \\
\left(u_{n}\left(t_{0}\right), \partial_{t} u_{n}\left(t_{0}\right)\right) & =\boldsymbol{u}_{0}
\end{aligned}\right.
$$

Using (A.5) and (A.6) one can show that there exists a unique maximal solution

$$
\boldsymbol{u}_{n} \in C\left(\left[T_{1, n}, T_{2, n}\right], X^{s} \times H^{s}\right), \quad T_{1, n}<t_{0}<T_{2, n}
$$

and

$$
T_{1, n}>-\infty \Rightarrow \lim _{t \rightarrow T_{1, n}}\left\|\boldsymbol{u}_{n}\right\|_{X^{s} \times H^{s}}=+\infty, \quad T_{2, n}<+\infty \Rightarrow \lim _{t \rightarrow T_{2, n}}\left\|\boldsymbol{u}_{n}\right\|_{X^{s} \times H^{s}}=+\infty .
$$

From (A.5) and the energy estimate we have

$$
\left\|\boldsymbol{u}_{n}(t)\right\|_{X^{s} \times H^{s}} \lesssim\left\|\boldsymbol{u}_{0}\right\|_{X^{s} \times H^{s}}+\left|\int_{t_{0}}^{t} f\left(\|\boldsymbol{u}(\tau)\|_{X^{s} \times H^{s}}\right) \mathrm{d} \tau\right|,
$$

with a constant independent of $n$. This implies that there exist $\widetilde{T}_{1}<t_{0}, \widetilde{T}_{2}>t_{0}$ and a constant $C_{1}$ independent of $n$ such that

$$
\left\|\boldsymbol{u}_{n}(t)\right\|_{X^{s} \times H^{s}} \leq C_{1} \quad \forall n, \forall t \in\left[\widetilde{T}_{1}, \widetilde{T}_{2}\right]
$$

(in particular $\widetilde{T}_{1} \geq \sup _{n} T_{1, n}$ and $\widetilde{T}_{2} \leq \inf _{n} T_{2, n}$ ).

Now we need to verify that

$$
\lim _{n \rightarrow+\infty}\left\|\boldsymbol{u}_{n}(t)-\boldsymbol{u}(t)\right\|_{X^{1} \times H^{1}}=0 \quad \forall t \in\left[\widetilde{T}_{1}, \widetilde{T}_{2}\right] .
$$

To this end, we notice that $\boldsymbol{u}_{n}-\boldsymbol{u}$ solves the Cauchy problem:

$$
\left\{\begin{aligned}
\left(\partial_{t t}-\Delta\right)\left(u_{n}-u\right) & =f_{n}\left(u_{n}\right)-f(u), \\
\left(u_{n}\left(t_{0}\right), \partial_{t} u_{n}\left(t_{0}\right)\right) & =0
\end{aligned}\right.
$$

Since $\|\boldsymbol{u}(t)\|_{X^{1} \times H^{1}}$ is bounded and $\left\|\boldsymbol{u}_{n}(t)\right\|_{X^{1} \times H^{1}}$ are uniformly bounded for $t \in\left[\widetilde{T}_{1}, \widetilde{T}_{2}\right]$, (A.1) and (A.3) imply that for $t \in\left[\widetilde{T}_{1}, \widetilde{T}_{2}\right]$ there holds

$\left\|f_{n}\left(u_{n}(t)\right)-f(u(t))\right\|_{H^{1}} \leq\left\|f_{n}\left(u_{n}(t)\right)-f_{n}(u(t))\right\|_{H^{1}}+\left\|f_{n}(u(t))-f(u(t))\right\|_{H^{1}} \lesssim\left\|u_{n}(t)-u(t)\right\|_{X^{1}}+c_{n}$,

which yields (A.11) by the energy estimate and the Gronwall inequality.

From (A.10) and (A.11) we deduce

$$
\|\boldsymbol{u}(t)\|_{X^{s} \times H^{s}} \leq C_{1}, \quad \forall t \in\left[\widetilde{T}_{1}, \widetilde{T}_{2}\right] .
$$

The function $\boldsymbol{u}:\left[\widetilde{T}_{1}, \widetilde{T}_{2}\right] \rightarrow X^{s} \times H^{s}$ is weakly measurable (since it is measurable as a function to $\left.X^{1} \times H^{1}\right)$, hence it is measurable and $\boldsymbol{u} \in L^{\infty}\left(\left[\widetilde{T}_{1}, \widetilde{T}_{2}\right], X^{s} \times H^{s}\right)$. Using once again the energy estimate together with (A.4) it is easy to see that in fact $\boldsymbol{u} \in C\left(\left[\widetilde{T}_{1}, \widetilde{T}_{2}\right], X^{s} \times H^{s}\right)$. 


\section{REFERENCES}

[1] J. Bourgain and W. Wang. Construction of blowup solutions for the nonlinear Schrödinger equation with critical nonlinearity. Ann. Scuola Norm. Sup. Pisa Cl. Sci. (4), 25:197-215, 1997.

[2] S.-N. Chow and J. K. Hale. Methods of Bifurcation Theory, volume 251 of Grundlehren der mathematischen Wissenschaften. Springer, 1982.

[3] F. M. Christ and M. I. Weinstein. Dispersion of small amplitude solutions of the generalized Korteweg-de Vries equation. J. Funct. Anal., 100:87-109, 1991.

[4] R. Côte, C. Kenig, A. Lawrie, and W. Schlag. Profiles for the radial focusing 4d energy-critical wave equation. Preprint, 2014.

[5] R. Donninger and B. Schörkhuber. Stable blow up dynamics for energy supercritical wave equations. Trans. Amer. Math. Soc., 366(4):2167-2189, 2014.

[6] R. Donninger and B. Schörkhuber. Stable blowup for wave equations in odd space dimensions. Preprint, 2015.

[7] T. Duyckaerts, C. Kenig, and F. Merle. Universality of blow-up profile for small radial type II blow-up solutions of the energy-critical wave equation. J. Eur. Math. Soc., 13(3):533-599, 2011.

[8] T. Duyckaerts, C. Kenig, and F. Merle. Universality of the blow-up profile for small type II blow-up solutions of the energy-critical wave equation: the nonradial case. J. Eur. Math. Soc., 14(5):1389-1454, 2012.

[9] T. Duyckaerts, C. Kenig, and F. Merle. Classification of the radial solutions of the focusing, energy-critical wave equation. Camb. J. Math., 1(1):75-144, 2013.

[10] T. Duyckaerts and F. Merle. Dynamics of threshold solutions for energy-critical wave equation. Int. Math. Res. Pap. IMRP, 2008.

[11] J. Ginibre, A. Soffer, and G. Velo. The global cauchy problem for the critical nonlinear wave equation. J. Funct. Anal., 110:96-130, 1992.

[12] M. Hillairet and P. Raphaël. Smooth type II blow up solutions to the four dimensional energy critical wave equation. Analysis \& PDE, 5(4):777-829, 2012.

[13] J. Jendrej. Construction of type II blow-up solutions for the energy-critical wave equation in dimension 5. Preprint, 2015.

[14] C. E. Kenig and F. Merle. Global well-posedness, scattering and blow-up for the energy-critical focusing nonlinear wave equation. Acta Math., 201(2):147-212, 2008.

[15] J. Krieger, W. Schlag, and D. Tataru. Slow blow-up solutions for the $H^{1}\left(\mathbb{R}^{3}\right)$ critical focusing semilinear wave equation. Duke Math. J., 147(1):1-53, 2009.

[16] Y. Martel and F. Merle. Instability of solitons for the critical generalized Korteweg-de Vries equation. Geom. Funct. Anal., 11:74-123, 2001.

[17] Y. Martel, F. Merle, and P. Raphaël. Blow up for the critical gKdV equation I: dynamics near the soliton. Preprint, 2012.

[18] Y. Martel, F. Merle, and P. Raphaël. Blow up for the critical gKdV equation III: exotic regimes. Preprint, 2012.

[19] F. Merle and P. Raphaël. Profiles and quantization of the blow up mass for critical nonlinear Schrödinger equation. Commun. Math. Phys., 253(3):675-704, 2005.

[20] F. Merle and H. Zaag. Determination of the blow-up rate for the semilinear wave equation. Amer. J. Math., 125(5):1147-1164, 2003.

[21] F. Merle and H. Zaag. Determination of the blow-up rate for a critical semilinear wave equation. Math. Ann., $331(2): 395-416,2005$.

[22] J. Shatah and M. Struwe. Well-posedness in the energy space for semilinear wave equations with critical growth. Internat. Math. Res. Notices, 7:303-309, 1994.

École Polytechnique, CMls, 91128 Palaiseau, France

E-mail address: jacek.jendrej@polytechnique.edu 